\title{
Intense-terahertz-laser-modulated magnetopolaron effect on shallow-donor states in the presence of magnetic field in the Voigt configuration
}

\author{
Weiyang Wang,,$^{1,2, *}$ B. Van Duppen, ${ }^{2, \dagger}$ and F. M. Peeters ${ }^{3,2, \dagger}$ \\ ${ }^{1}$ School of Physics and Electronics Information, Shangrao Normal University, Shangrao, Jiangxi 334001, China \\ ${ }^{2}$ Departement Fysica, Universiteit Antwerpen, Groenenborgerlaan 171, B-2020 Antwerpen, Belgium \\ ${ }^{3}$ School of Physics and Astronomy and Yunnan Key Laboratory for Quantum Information, Yunnan University, Kunming 650091, China
}

(Received 24 October 2018; revised manuscript received 16 January 2019; published 29 January 2019)

\begin{abstract}
The laser-modulated magnetopolaron effect on shallow donors in semiconductors is investigated in the presence of a magnetic field in the Voigt configuration. A nonperturbative approach is used to describe the electron-photon interaction by including the radiation field in an exact way via a laser-dressed interaction potential. Through a variational approach we evaluate the donor binding energy. We find that the interaction strength of the laser-dressed Coulomb potential in the $z$ direction cannot only be enhanced but also weakened by the radiation field, while that in the $x-y$ plane is only weakened. In this way, the binding energy of the states with odd $z$ parity, like $2 p_{z}$, can be decreased or increased with respect to its static binding energy by the radiation field, while that of the other states can be only decreased. Furthermore, all binding energies become insensitive to the magnetic field if the radiation field is strong. The magnetopolaron effect on these energies is studied within second-order time-dependent perturbation theory. In the nonresonant region, a laser-modulated magnetopolaron correction, including the effect of single-photon processes, is observed. In the resonant region, a laser-modulated magnetopolaron effect, accompanied by the emission and absorption of a single photon, is found. Moreover, the $1 s \rightarrow 2 p^{+}$transition, accompanied by the emission of a single photon, is tuned by the radiation field into resonance with the longitudinal-optical phonon branch. This is electrically analogous to the magnetopolaron effect, and therefore we name it the dynamical magnetopolaron effect. Finally, by changing the frequency of the radiation field, these interesting effects can be tuned to be far away from the reststrahlen band and, therefore, can be detected experimentally. This in turn provides a direct measure of the electron-phonon interaction.
\end{abstract}

DOI: 10.1103/PhysRevB.99.014114

\section{INTRODUCTION}

The interaction between electrons and longitudinal-optical (LO) phonons is an important mechanism in polar semiconductors and related nanostructures that limits the mobility of electrons, renormalizes the electron mass, and results in intriguing resonant phenomena. Particular interest has been paid to the electron-LO-phonon interaction in polar semiconductor systems when subjected to a strong magnetic field [1], which is able to tune, e.g., shallow impurity levels into resonance. Because of the single-particle nature of the shallow impurity system, possible complications arising from manybody effects such as screening, interface, and Pauli exclusion effects [2] can be safely neglected in the interpretation of the observed phenomena. The consequences of electron-LOphonon interaction become particularly apparent when the electronic transition associated with shallow-impurity states is tuned by the magnetic field into resonance with the optical modes, resulting in a resonant splitting of this transition. As a result, a characteristic anticrossing behavior, the so-called magnetopolaron effect (MPE), is clearly observed. This effect has been extensively studied experimentally and theoretically

\footnotetext{
*wang.weiyang.cmt@foxmail.com

†ben.vanduppen@uantwerpen.be

${ }^{\ddagger}$ francois.peeters@uantwerpen.be
}

for several decades in bulk semiconductors [3-5] as well as in related low-dimensional systems including quantum wells (QWs) [6], superlattices [7], and quantum dots (QDs) [8]. Most importantly, the resonant splitting of shallow-impurity states resulting from the resonant interaction with the LO phonon, in turn, provides a direct way to measure the strength of the electron-phonon (e-p) interaction.

In classical far-infrared experiments, the intra-impurity $1 s \rightarrow 2 p^{+}$transition energy has been tuned into resonance with the LO phonon energy using a high magnetic field [3,4]. In semiconductors having large effective electron mass and high LO phonon energy, however, it is difficult to observe a MPE in the $1 s \rightarrow 2 p^{+}$transition because it requires extreme high magnetic field, especially for the case of $\mathrm{GaN}$ that requires around $200 \mathrm{~T}$ to observe a MPE [5]. Moreover, the resonant polaron splitting in weakly polar semiconductors is small and, therefore, is difficult to observe a MPE on shallow-impurity states due to the reststrahlen band $[3,4,6,7]$. Therefore, it is desirable to search for new ways to measure the MPE on shallow-impurity states in low magnetic field and away from the reststrahlen band.

With the advent of high-power, tunable, and linearly polarized laser sources such as $\mathrm{CO}_{2}$ and free-electron lasers (FELs), new possibilities have arisen in studying the behavior of shallow-impurity states in semiconductors and related nanostructures when irradiated by an intense terahertz (THz) laser field (ITLF) $[9,10]$. As a consequence, some 
important and distinctive phenomena associated with shallowimpurity states in laser-driven semiconductor systems have been observed theoretically and experimentally, such as the dichotomy of a hydrogen atom [11] and exciton [12], the stabilization of atomic hydrogen [13] and shallow impurity [14] as well as exciton [15], an increase of positronium lifetime [16], and a rapid decrease of the binding energy with increasing laser field intensity in semiconductors $[14,17]$ while a red- or blueshift in binding energy with respect to its static binding energy in semiconductor nanostructures depending on its geometric parameters [18-20]. The ITLF effects on shallow-impurity states can be detected experimentally, for instance, by measuring the changes in the binding energy and intra-impurity transition energy, which show up as modification of the optical properties in laser-driven semiconductor systems. This is why the behavior of shallow-impurity states in laser-driven semiconductor systems has been widely studies in the last few decades.

Recently, it has been found that the ITLF effects on the intra-impurity state transition in semiconductor nanostructures can be as important as the effect of a magnetic field [19]. As a result, the study of the nonlinear magneto-optical properties of shallow-impurity states in bulk semiconductors [21] as well as in low-dimensional systems including QWs [22], quantum well wires [23], and QDs [24] under magnetic and ITLFs has been of tremendous interest due to their promising applications in designing new efficient optoelectronic devices manipulated by the two external fields [25]. These studies show that the magneto-optical properties of shallow-impurity states in laser-driven semiconductor systems are strongly affected, not only by the geometric parameters, but also by the magnetic field and ITLF through the laser-dressed potential. This indicates that the two external fields can be properly chosen, together with an appropriate choice of geometric parameters, to produce desired physical properties of shallow-impurity states in such laser-driven semiconductor systems. On the other hand, the e-p interaction in semiconductors and related nanostructures subjected simultaneously to magnetic field and ITLF can be tuned by these external fields. This mechanism, however, becomes suppressed when the ITLF is intense enough, meanwhile the effective electron-photon interaction is enhanced [26,27]. Therefore, it is expected that laser-modulated MPE on shallow-impurity states are experimentally observable in semiconductors and related nanostructures under magnetic and ITLFs. In our recent work we proposed a nonperturbative approach to investigate the MPE on shallow-donor states in GaAs when irradiated by ITLF within the Faraday configuration, where we find that not only the donor binding energy but also the MPE are appropriately adjusted by the two external fields [28].

The present work is concerned with the magneto-optical properties of shallow-donor states in semiconductors under magnetic and ITLFs within the Voigt configuration [3]. We use the nonperturbative approach in combination with a variational approach to investigate the effect of the two external fields on the donor binding energy. Thus the laser-modulated MPE is studied using time-dependent perturbation theory (TdPT). This paper is organized as follows. In Sec. II we outline the nonperturbative approach together with the TdPT.

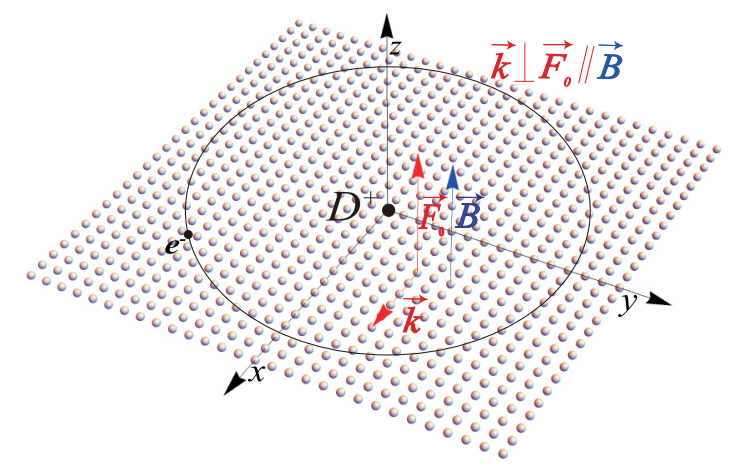

FIG. 1. Schematic representation of a shallow donor $D^{+}$in a bulk semiconductor in the presence of ITLF and magnetic field within the Voigt configuration. Here $\vec{k}$ is the wave vector of the applied ITLF.

Section III presents our numerical results and discussions, and concluding remarks are given in Sec. IV.

\section{THEORETICAL APPROACH}

\section{A. Electronic states}

We consider the situation where a static magnetic field $\vec{B}=(0,0, B)$, with vector potential $\vec{A}_{B}=\frac{1}{2}[\vec{B} \times \vec{R}]$, is applied along $z$ direction of a bulk semiconductor, meanwhile, an ITLF is applied along the $x$ direction and linearly polarized parallel to the magnetic field, as shown in Fig. 1. Under the usual dipole approximation, the vector potential induced by the ITLF is given as $\vec{A}_{t}=\left(F_{0} / \omega\right) \cos (\omega t) \vec{e}_{z}$ and expressed in the Coulomb gauge with $F_{0}$ and $\omega$ being the amplitude and angular frequency of the laser field, respectively. Within the effective-mass approximation, the time-dependent Hamiltonian of a shallow-donor impurity in this laser-driven semiconductor system is given, in the absence of e-p interaction, by

$$
H_{e}(\vec{R}, t)=\frac{1}{2 m^{*}}(\vec{p}+e \vec{A})^{2}+V(\vec{R}),
$$

where $m^{*}$ is the electron effective mass, $\vec{A}=\vec{A}_{t}+\vec{A}_{B}, \vec{p}=$ $-i \hbar \nabla$ and $\vec{R}$ are the momentum and position operators of the electron, respectively, and $V(\vec{R})=-e^{2} / \epsilon|\vec{R}|$ is the Coulomb potential induced by the electron-impurity interaction with $\epsilon$ being the dielectric constant of the medium.

Due to the time-dependent feature of the Hamiltonian for describing such a electron-impurity system as given in Eq. (1), in principle, we have to make use of a nonperturbative approach $[21,28-30]$ to seek the solution of the corresponding time-dependent Schrödinger equation, namely,

$$
i \hbar \frac{\partial \Psi(\vec{R}, t)}{\partial t}=H_{e}(\vec{R}, t) \Psi(\vec{R}, t) .
$$

As in the Voigt configuration the two external fields are not directly coupled with each other, the time-dependent unitary transformation proposed in Refs. [21,28-30] is redefined as

$$
U=\exp \left(\frac{i}{\hbar} f_{t}\right) \exp \left(\frac{i}{\hbar} \alpha_{t} p_{z}\right) \exp \left(\frac{i}{\hbar} v_{t} z\right),
$$

where $p_{z}=-i \hbar \partial / \partial z$ is the momentum operator along the $z$ direction, $f_{t}$ is an arbitrary time-dependent function, and 
$\alpha_{t}$ and $v_{t}$ are the coordinate and phase shifts, respectively. By performing this transformation on Eq. (2) and making use of the arbitrariness of $\alpha_{t}, v_{t}$, and $f_{t}$ as the initial conditions [30,31], one obtains

$$
i \hbar \frac{\partial \psi(\vec{R}, t)}{\partial t}=\widetilde{H}_{e}(\vec{R}, t) \psi(\vec{R}, t),
$$

where

$$
\widetilde{H}_{e}(\vec{R}, t)=\frac{1}{2 m^{*}}\left(\vec{p}+e \vec{A}_{B}\right)^{2}+V\left(\vec{R}+\vec{\alpha}_{t}\right) .
$$

Here $\vec{\alpha}_{t}=\alpha_{0} \sin (\omega t) \vec{e}_{z}$ describes the quiver motion of a classical electron, with $\alpha_{0}=e F_{0} /\left(m^{*} \omega^{2}\right)$ being the laser-dressing parameter. It is apparent that $\vec{\alpha}_{t}$ is identical to that only under an ITLF $[14,32]$ but is quite different from that in the Faraday configuration [28], since there is no direct coupling between the two external fields in the Voigt configuration. Moreover, Eq. (4) is eminently suitable in describing the electronic states subjected simultaneously to two external applied fields since the time dependence has been transferred from the momentum term to the potentials associated with laser-driven semiconductor systems, which is completely equivalent to Eq. (2). In the calculation we have used $\alpha_{t}, v_{t}$, and $f_{t}$ to cancel the time-dependent terms in $\widetilde{H}_{e}(\vec{R}, t)$ that are linear in $\vec{p}$ and $\vec{R}$ and the terms that depend only on time [30]. Note that such initial conditions have been widely and successfully used in solving time-dependent problems [28,31]. Moreover, no assumptions concerning the validity of Eqs. (3)-(5) have been made so far, so that the unitary transformation defined in Eq. (3) can be used either as an intense or a weak laser field in the Voigt configuration.

In Eq. (5), the time-dependent laser-dressed Coulomb potential ( $\mathrm{LdCP})$ is obtained by

$$
V\left(\vec{R}+\vec{\alpha}_{t}\right)=-\frac{e^{2}}{\epsilon\left(R^{2}+a^{2}\right)^{1 / 2}}\left(1-b_{t}\right)^{-1 / 2},
$$

where $b_{t}=\left[a^{2} \cos (2 \omega t)-2 \vec{R} \cdot \vec{\alpha}_{t}\right] /\left(R^{2}+a^{2}\right)$ with $a=$ $\alpha_{0} / \sqrt{2}$. According to the previous approximation [33] widely used in studying the variation characteristics of shallow-impurity states under ITLFs [34,35], Eq. (6) can be simplified as a time-independent one which is essentially the time averaged of the LdCP, namely,

$$
V(R, a)=-\frac{e^{2}}{\epsilon\left(R^{2}+a^{2}\right)^{1 / 2}}\left[1+\frac{3}{16} \frac{8 z^{2} a^{2}+a^{4}}{\left(R^{2}+a^{2}\right)^{2}}\right],
$$

which is identical to the well-known result obtained previously for electrons bound on liquid helium [36]. Different from the LdCP obtained in the Faraday configuration [28], this LdCP is only affected by the ITLFs. In the absence of the ITLFs, the LdCP reduces to the bare Coulomb potential. In contrast, in the opposite limit of high laser-dressing parameter the LdCP approaches zero, implying that the ITLF effects are to produce a laser-modulated Coulomb potential such that the donor states can be effectively tuned by the ITLFs. Moreover, it has been shown that this approximation is valid for the description of a shallow impurity under either an intense or weak laser field [28,33-36]. In addition, $v_{t}=0$ and $f_{t}=$ $-E_{e m}[t+\sin (2 \omega t) /(2 \omega)]$ with $E_{e m}=e^{2} F_{0}^{2} / 4 m^{*} \omega^{2}$ being the ponderomotive energy [14] induced by the radiation field. Interestingly, $E_{e m}$ will lead to a blueshift in the fundamental absorption edge known as dynamical Franz-Keldysh effect (DFKE) [37] in laser-driven semiconductor systems.

By substituting Eq. (7) into Eq. (5), one may straightforwardly obtain a time-independent Hamiltonian in the accelerated frame of reference, namely,

$$
\widetilde{H}_{e}(\vec{R})=\frac{1}{2 m^{*}}\left(\vec{p}+e \vec{A}_{B}\right)^{2}+V(R, a),
$$

which is identical to that of the hydrogenic system under a static magnetic field [3,4] or two external fields in the Faraday configuration [28], except for the LdCP term $V(R, a)$. It is obvious that the Schrödinger equation corresponding to $\widetilde{H}_{e}(\vec{R})$ cannot be solved exactly, so that we rely on a variational calculation for the donor states. In this way we adopt the same trial wave functions as used in Refs. [3,4,28], namely,

$$
\psi_{n m p}(\rho, \phi, z)=\rho^{|m|} z^{p} e^{i m \phi} e^{-\xi \rho^{2}-\zeta R}\left(1-\lambda R_{\sigma}\right),
$$

where $R_{\sigma}=\left(\rho^{2}+\sigma^{2} z^{2}\right)^{1 / 2}$ and $\lambda$ is nonzero only for the $2 s$ state and is determined such that this state is orthogonal to the ground state. The variational parameters $\xi, \zeta$, and $\sigma$ are determined such that they minimize the unperturbed energy

$$
E_{n m p}^{(0)}=\frac{\left\langle\psi_{n m p}\left|\widetilde{H}_{e}(\vec{R})\right| \psi_{n m p}\right\rangle}{\left\langle\psi_{n m p} \mid \psi_{n m p}\right\rangle} .
$$

In this study, we limit ourselves to those donor states that are most likely to be observed in experiments [3-5], so that it is sufficient to include the following states in the calculations: $1 s=|1,0,0\rangle, 2 s=|2,0,0\rangle, 2 p_{z}=|2,0,1\rangle, 2 p^{ \pm}=$ $|2, \pm 1,0\rangle, 3 d^{-2}=|3,-2,0\rangle$, and $4 f^{-3}=|4,-3,0\rangle$. In addition, the binding energy of the $i$ th $[i=(n, m, p)]$ donor state is defined by

$$
E_{i}^{b}=E_{N}-E_{i}^{(0)},
$$

where $E_{N}=(N+1 / 2) \hbar \omega_{c}$ is the $N$ th Landau level (LL) energy with $N=1$ for $i=2 p^{+}, N=0$ for the rest of the considered donor states, and $\omega_{c}=e B / m^{*}$ being the cyclotron frequency for a free electron.

\section{B. Magnetopolaron correction}

In the accelerated frame of reference, the Fröhlich Hamiltonian $H_{e p}$ for e-p coupling is converted into a laser-dressed oscillating potential (LdOP) $[27,28,38]$. The Hamiltonian $H_{e p}$ can be cast into a suitable form through a unitary transformation $U^{\dagger} H_{e p}(\vec{R}) U$, namely,

$$
\widetilde{H}_{e p}(\vec{R}, t)=\sum_{\vec{Q}, n}\left[\widetilde{V}_{\vec{Q}} a_{\vec{Q}} e^{i(\vec{Q} \cdot \vec{R}+n \theta)}+\widetilde{V}_{\vec{Q}}^{*} a_{\vec{Q}}^{\dagger} e^{-i(\vec{Q} \cdot \vec{R}+n \theta)}\right],
$$

where $\left(a_{\vec{Q}}, a_{\vec{Q}}^{\dagger}\right)$ are the annihilation and creation operators of a LO phonon with wave vector $\vec{Q}=\left(\vec{q}, q_{z}\right), \theta=\omega t$, and $\widetilde{V}_{\vec{Q}}=$ $J_{n}\left(q_{z} \alpha_{0}\right) V_{\vec{Q}}$ is the laser-modulated e-p coupling [28,38] with $J_{n}(x)$ being the Bessel function of the first kind and $\left|V_{\vec{Q}}\right|^{2}=$ $4 \pi \alpha_{\mathrm{LO}} L_{0}\left(\hbar \omega_{\vec{Q}}\right)^{2} / Q^{2} V$ with $L_{0}$ being the polaron radius, $\alpha_{\mathrm{LO}}$ being the standard Fröhlich coupling constant, $V$ being the volume of the system, and $\omega_{\vec{Q}}$ being the LO phonon frequency. Obviously the LdOP depends on time and thus cannot produce a stationary e-p coupling, which is effectively adjusted by the ITLFs via the Bessel function, leading to a decreasing 
behavior of the LdOP with increasing $\alpha_{0}$. In the absence of the ITLFs (i.e., $F_{0} \rightarrow 0$ ), due to $\lim _{x \rightarrow 0} J_{n}(x)=\delta_{n, 0}$, we have

$$
H_{e p}(\vec{R})=\sum_{\vec{Q}}\left[V_{\vec{Q}} a_{\vec{Q}} e^{i \vec{Q} \cdot \vec{R}}+V_{\vec{Q}}^{*} a_{\vec{Q}}^{\dagger} e^{-i \vec{Q} \cdot \vec{R}}\right],
$$

which is the well-known LO phonon Hamiltonian in the fieldfree case [4,27].

The energy shift of the $i$ th donor state in a weakly polar semiconductor due to the LdOP for e-p interaction is, within second order TdPT [39], given by [28]

$$
\Delta E_{i}=-\sum_{j, n^{\prime}} \sum_{\vec{Q}} \frac{\left|\left\langle\psi_{j} ; \vec{Q}\left|H_{e p}(\vec{R})\right| \psi_{i} ; \overrightarrow{0}\right\rangle\right|^{2} J_{n^{\prime}}^{2}\left(q_{z} \alpha_{0}\right)}{\hbar \omega_{\vec{Q}}+n^{\prime} \hbar \omega+E_{j}^{(0)}-E_{i}^{(0)}-\Delta_{i}},
$$

where $\Delta_{i}=0$ for all donor states in the polaron nonresonant region which corresponds to Rayleigh-Schrödinger perturbation theory (RSPT), and $\Delta_{2 p^{+}}=\Delta E_{2 p^{+}}-\Delta E_{1 s}$ for the $2 p^{+}$state in the polaron resonant region that corresponds to improved Wigner-Brillouin perturbation theory (IWBPT) $[3,4,6-8]$. Note that we have successfully applied the TdPT to study the MPE on the donor states under two external fields in the Faraday configuration recently [28]. In Eq. (14), $\left|\psi_{j} ; \vec{Q}\right\rangle$ characterizes a state that consists of an electron with unperturbed energy $E_{j}^{(0)}$ and a LO phonon with momentum $\hbar \vec{Q}$ and energy $\hbar \omega_{\vec{Q}}$, and $n^{\prime}$ refers to emission and absorption of photons with energy $n^{\prime} \hbar \omega$ switched by the Bessel function. In the absence of the ITLFs (i.e., $F_{0} \rightarrow 0$ ), due to $\lim _{x \rightarrow 0} J_{n^{\prime}}(x)=\delta_{n^{\prime}, 0}$, the magnetopolaron correction (MPC) $\Delta E_{i}$ obtained in Eq. (14) reduces to the well-known MPC previously obtained by using IWBPT derived in the absence of radiation field [3,4,6-8].

It is possible to calculate Eq. (14) approximately and to carry out the sum $\sum_{j}$ formally in such a way that we only need to include a few relevant donor states to calculate the polaron shift to the donor energy levels. Following the procedure described in Ref. [40], Eq. (14) can be cast into a simple and practical expression

$$
\begin{aligned}
\Delta E_{i}= & -\alpha_{\mathrm{LO}} \hbar \omega_{\mathrm{LO}}-\sum_{j, n^{\prime}} \sum_{\vec{Q}}\left|\left\langle\psi_{j} ; \vec{Q}\left|H_{e p}(\vec{R})\right| \psi_{i} ; \overrightarrow{0}\right\rangle\right|^{2} \\
& \times \frac{J_{n^{\prime}}^{2}\left(q_{z} \alpha_{0}\right)\left(E_{i}^{(0)}+\Delta_{i}+Q^{2}-E_{j}^{(0)}-n^{\prime} \hbar \omega\right)^{2}}{\left(\hbar \omega_{\mathrm{LO}}+Q^{2}\right)^{2}\left(\hbar \omega_{\mathrm{LO}}+n^{\prime} \hbar \omega+E_{j}^{(0)}-E_{i}^{(0)}-\Delta_{i}\right)},
\end{aligned}
$$

which is composed of two terms: (i) the first $-\alpha_{\mathrm{LO}} \hbar \omega_{\mathrm{LO}}$ is the polaron correction of a free electron in the field-free case; and (ii) the second term results from the contribution of the two external fields and depends on the specific donor states. For these reasons we only need to evaluate the second term on the right-hand side of Eq. (15) to calculate the MPC for the donor states. Notice that we have already adopted a wavevector-independent phonon frequency, say $\omega_{\vec{Q}}=\omega_{\mathrm{LO}}$, in the calculation.

According to Refs. $[3,6-8,28]$, the MPC in the polaron nonresonant region is given by

$$
\Delta E_{i}=-\alpha_{\mathrm{LO}}\left[\hbar \omega_{\mathrm{LO}}+\frac{2}{3} \frac{\left\langle\psi_{i}\left|\left(\vec{p}+e \vec{A}_{B}\right)^{2}\right| \psi_{i}\right\rangle}{2 m^{*}\left\langle\psi_{i} \mid \psi_{i}\right\rangle}\right],
$$

which is in line with previous results $[3,6-8]$ obtained for donor states in the absence of a radiation field, indicating that photon processes do not contribute to the MPC in this region. It has been shown that Eq. (16) is a good approximation to Eq. (15) for all considered donor states under two arbitrary external fields [28], except for the $2 p^{+}$state. For the $2 p^{+}$ state we follow Refs. $[3,6-8,28]$, then the MPC is given, in the vicinity of the polaron resonant region, by

$$
\begin{aligned}
\Delta E_{2 p^{+}}= & \Delta E_{2 p^{-}}-\sum_{i=2 p^{ \pm}, j} \sum_{\vec{Q}} m_{i} \frac{\left|\left\langle\psi_{j} ; \vec{Q}\left|H_{e p}(\vec{R})\right| \psi_{i} ; \overrightarrow{0}\right\rangle\right|^{2}}{\left(\hbar \omega_{\mathrm{LO}}+Q^{2}\right)^{2}} \\
& \times \sum_{n^{\prime}} \frac{\left(E_{j}^{(0)}+n^{\prime} \hbar \omega-E_{i}^{(0)}-Q^{2}\right)^{2} J_{n^{\prime}}^{2}\left(q_{z} \alpha_{0}\right)}{\left(\hbar \omega_{\mathrm{LO}}+n^{\prime} \hbar \omega+E_{j}^{(0)}-E_{i}^{(0)}-\Delta_{i}\right)},
\end{aligned}
$$

where $m_{i}$ is the magnetic quantum number of the $i$ th state, $\Delta E_{2 p^{-}}$is the nonresonant MPC calculated from Eq. (16), and the sum $\sum_{j}$ includes only the relevant states. Distinct from Eq. (16), the photon processes have considerable effect on the MPC of the $2 p^{+}$state in the polaron resonant region.

Finally, the effect of band nonparabolicity on the donor states is taken into account by using the standard Kane model [41]

$$
E_{\mathrm{np}}=\frac{E_{g}}{2}\left[-1+\left(1+4 \frac{E_{p}}{E_{g}}\right)^{1 / 2}\right],
$$

where $E_{p}$ and $E_{\mathrm{np}}$ are the donor energy levels with and without the effect of band nonparabolicity, respectively, and $E_{g}$ is the energy gap of the material. This model has been applied to successfully describe band nonparabolicity for the donor transition energies in bulk GaAs $[3,28]$.

\section{RESULTS AND DISCUSSIONS}

The numerical results of this paper pertain to a shallow donor in GaAs bulk semiconductor. In the calculations the material parameters are taken as (i) effective-electron mass ratio $m^{*} / m_{e}=0.067$, with $m_{e}$ being the free-electron mass; (ii) high-frequency dielectric constant $\varepsilon=10.9$ due to the presence of ITLFs; (iii) LO-phonon energy $\hbar \omega_{\mathrm{LO}}=$ $36.75 \mathrm{meV}$; (iv) electron-LO-phonon coupling constant $\alpha_{\mathrm{LO}}=0.068$; and (v) band gap $E_{g}=1.52 \mathrm{eV}$. For convenience we introduce dimensionless units expressed in terms of the Bohr radius $a^{*}=\hbar^{2} \epsilon / m^{*} e^{2}=8.61 \mathrm{~nm}$, the Rydgerg energy $R^{*}=\left(\hbar / a^{*}\right)^{2} / 2 m^{*}=7.67 \mathrm{meV}$, and the dimensionless parameter $\gamma=\left(a^{*} / l_{B}\right)^{2}$ for magnetic-field strength with magnetic length $l_{B}=(\hbar / e B)^{1 / 2}$.

This section is organized as follows. In Secs. III A and III B we, respectively, outline the ITLF and magnetic field effects on the donor binding energy and explain the related physical mechanisms. The laser-modulated MPC with considering optical channels to the donor energy levels is calculated in Sec. IIIC. The laser-modulated MPE accompanied by emission and absorption of a single photon is discussed in Sec. III D and the dynamical magnetopolaron effect is presented in Sec. III E. 

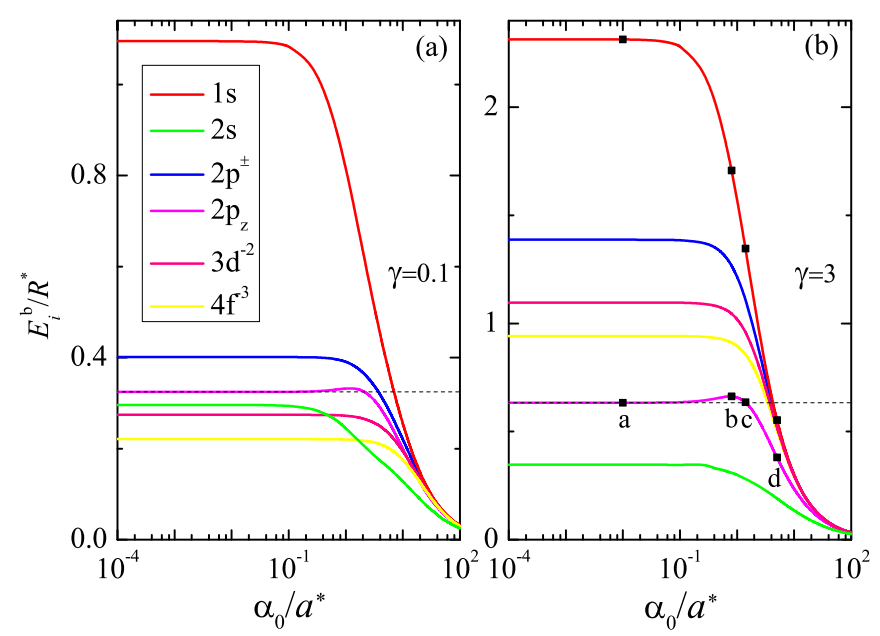

FIG. 2. The dependence of the binding energy for the few lowest states as a function of the laser-dressing parameter $\alpha_{0}$ at fixed $\gamma=0.1$ (left figure) and $\gamma=3.0$ (right figure). Here the horizontal dashed lines represent the static donor binding energy in the absence of the ITLFs.

\section{A. ITLF effect on the donor binding energy}

A careful analysis of Eq. (8) reveals that the influence of ITLFs on the donor states is through the laser-dressing parameter $\alpha_{0}=e F_{0} /\left(m^{*} \omega^{2}\right) \propto F_{0} / \omega^{2}$. This indicates that the laser field intensity and frequency affect the donor binding energy in the opposite way. As a result, we only need to investigate the dependence of the donor binding energy on $\alpha_{0}$. The results are shown in Fig. 2. For comparison, the static results corresponding to the absence of an ITLF are also shown by the horizontal dashed lines.

It is apparent that the binding energy is the static binding energy in the low- $\alpha_{0}$ limit. Then, as $\alpha_{0}$ increases, the binding energy of the $2 p_{z}$ states increases slightly at first, and after reaching a maximum, the binding energy rapidly decreases. This behavior is similar to that in the Faraday configuration [28]. In contrast, the binding energy of the other states monotonically decreases with increasing $\alpha_{0}$, which is similar to that found in the presence of the ITLFs [14,17]. Finally, the binding energy of all donor states approaches zero in the high- $\alpha_{0}$ limit, indicating that the shallow donor is ionized. This is diametrically opposed to the previous results with only ITLFs where the state remains stable $[14,17]$.

In order to understand the behavior of the donor binding energy in the ITLFs, we calculate the effective width of the wave functions of the donor states. Following the method described in Ref. [42], the effective widths in the $x-y$ plane and in the $z$ direction are, respectively, given by $r_{\rho}=\left[\left\langle\rho^{2}\right\rangle / 2\right]^{1 / 2}$ and $r_{z}=\left[\left\langle z^{2}+\alpha_{0}^{2}\right\rangle\right]^{1 / 2}$. The results for $r_{\rho}$ and $r_{z}$ associated with the $1 s$ and $2 p_{z}$ states are shown in Fig. 3, where the points $a, b, c$, and $d$ correspond to those indicated in Fig. 2(b) by the square symbols. For comparison, the static results related to the absence of an ITLF are also displayed by horizontal dashed lines. Here we do not plot the effective width related to the other states since the behavior of the binding energy for these states in the ITLFs is similar to that of the ground state as shown in Fig. 2.
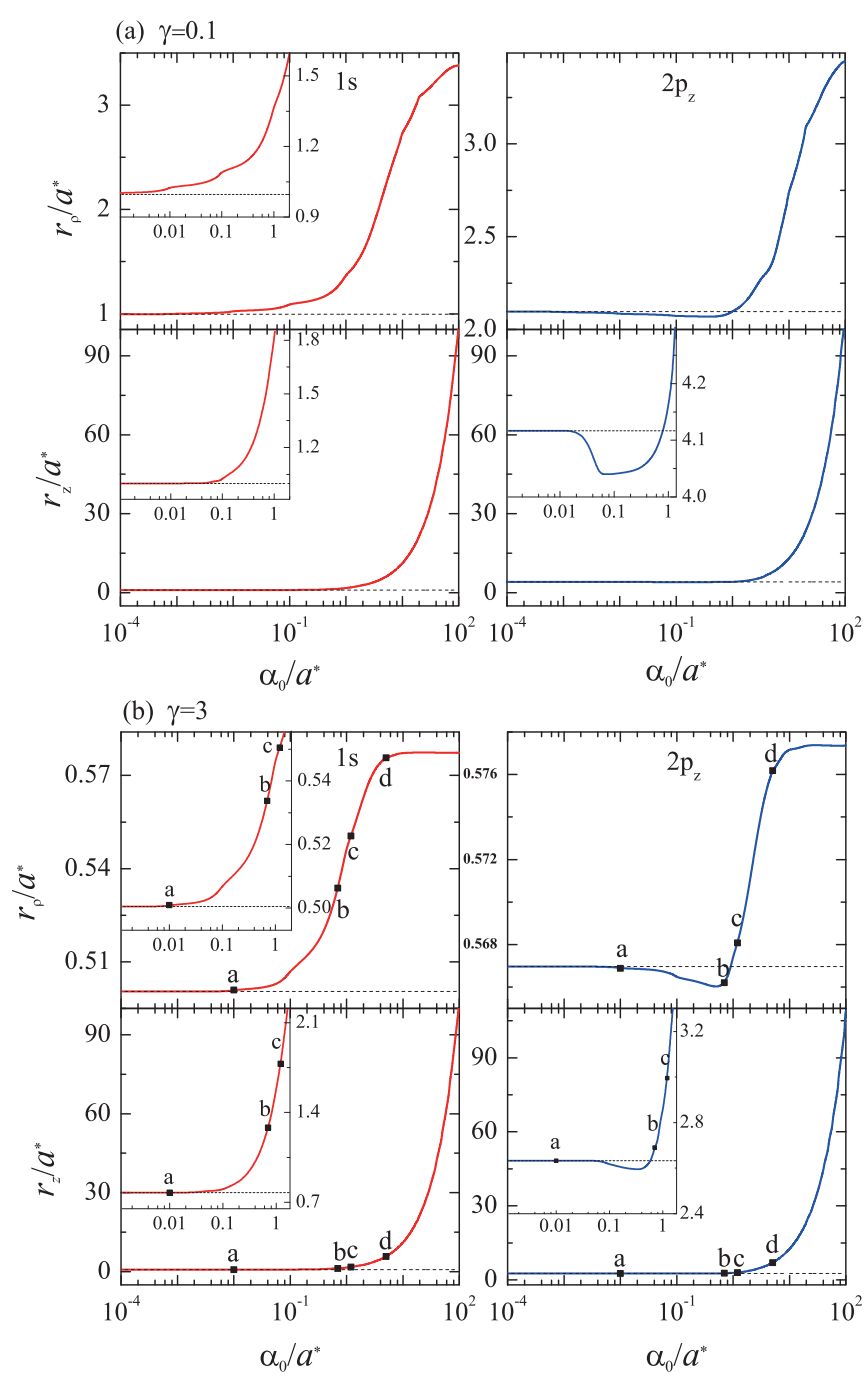

FIG. 3. The effective width of the wave functions for the $1 s$ and $2 p_{z}$ states in the $x-y$ plane and in the $z$ direction as a function of $\alpha_{0}$ at fixed $\gamma$ as indicated. The horizontal dashed lines represent the static width in the absence of the ITLFs. Inset: The region with small width are enlarged. In (b) points $a, b, c$, and $d$ correspond to those indicated in Fig. 2(b).

From Fig. 3 it is clear that both $r_{\rho}$ and $r_{z}$ of the $1 s$ and $2 p_{z}$ states remains constant in the low- $\alpha_{0}$ limit, suggesting that the ITLF effect on these two states can be neglected, in agreement with Fig. 2. After that, both $r_{\rho}$ and $r_{z}$ of the $1 s$ state increase exponentially with increasing $\alpha_{0}$, suggesting a decrease of the impurity-electron localization, which leads to a decrease of the donor binding energy. In contrast, both $r_{\rho}$ and $r_{z}$ of the $2 p_{z}$ state considerably decrease at first, but after they reach their minima, $r_{\rho}$ and $r_{z}$ exponentially increase with increasing $\alpha_{0}$. This suggests that the impurity-electron localization of the $2 p_{z}$ state first increases appreciably with increasing $\alpha_{0}$, but after a critical value of $\alpha_{0}$, it decreases rapidly with $\alpha_{0}$, resulting in a similar variation of the binding energy with $\alpha_{0}$ as shown in Fig. 2. In addition, the width $r_{\rho}$ for both $1 s$ and $2 p_{z}$ states becomes independent on the laser-dressing parameter $\alpha_{0}$ in the high- $\alpha_{0}$ limit when $\gamma=3$, which is a result of the fact that the LdCP approaches zero in this case as reflected in Eq. (7) 

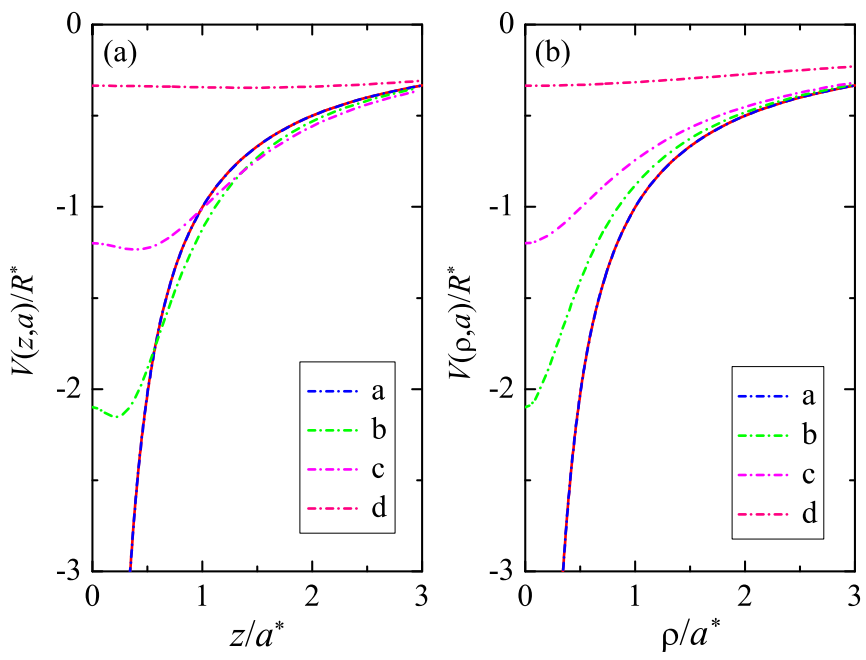

FIG. 4. Comparison of the bare Coulomb potential (red solid curve) with the LdCP for a shallow donor at fixed magnetic field $\gamma=3$ in the (a) $z$ direction and in the (b) $x-y$ plane. In (a) and (b) $a$, $b, c$, and $d$ correspond to the points indicated in Fig. 2(b).

such that the ITLF effect on the wave function of the shallow donor can be neglected.

Figure 3 also clearly shows that $r_{z}$ is significantly larger than $r_{\rho}$ for both $1 s$ and $2 p_{z}$ states, particularly in the high- $\alpha_{0}$ limit, which indicates that the donor states in the $x-y$ plane are more localized than that in the $z$ direction. As a result, the impurity-electron localization of the donor states in the $x-y$ plane plays a crucial role in the determination of the binding energy, which is evident by the fact that only the variation of the $r_{\rho}$ for the $2 p_{z}$ state with $\alpha_{0}$ is physically consistent with the dependence of its binding energy on $\alpha_{0}$, as shown in Fig. 3(b). The reason is that the magnetic field produces an additional lateral electronic confinement in the $x-y$ plane, leading to a more remarkable reduction in $r_{\rho}$ than in $r_{z}$. Meanwhile the ITLF has a direct effect on $r_{z}$ but an indirect effect on $r_{\rho}$, resulting in a more considerable increase in $r_{z}$ than in $r_{\rho}$. Consequently, $r_{z}$ becomes much larger than $r_{\rho}$. In addition, $r_{z}$ for the $2 p_{z}$ state does not reach its minimum at point $b$ and does not go back to the static width at point $c$ as shown in Fig. 3(b), which is due to the fact that $r_{z}$ is directly affected by the ITLF and thus rapidly increases with increasing $\alpha_{0}$ since $r_{z} \propto \alpha_{0}$.

To provide insights into the novel behaviors of the width observed in Fig. 3, we investigate how the ITLFs modulate the LdCP and, therefore modulate the width and, finally, tunes the donor binding energy. For simplicity, here we consider two special cases: $V(z, a)$ and $V(\rho, a)$, respectively, in the $z$ direction and in the $x-y$ plane. The results for $V(z, a)$ and $V(\rho, a)$ at fixed magnetic field $\gamma=3$ are shown in Fig. 4, where $a, b, c$, and $d$ correspond to the points indicated in Fig. 2(b).

Figure 4 clearly shows that the influence of the ITLF on the LdCP can be neglected when $\alpha_{0}$ is less than or equal to that at point $a$, in which the $\mathrm{LdCP}$ is completely equivalent to the bare Coulomb potential. In this case, it results in a static width as shown in Fig. 3(b) and, consequently, in a static binding energy as shown in Fig. 2(b). In Fig. 4(a),

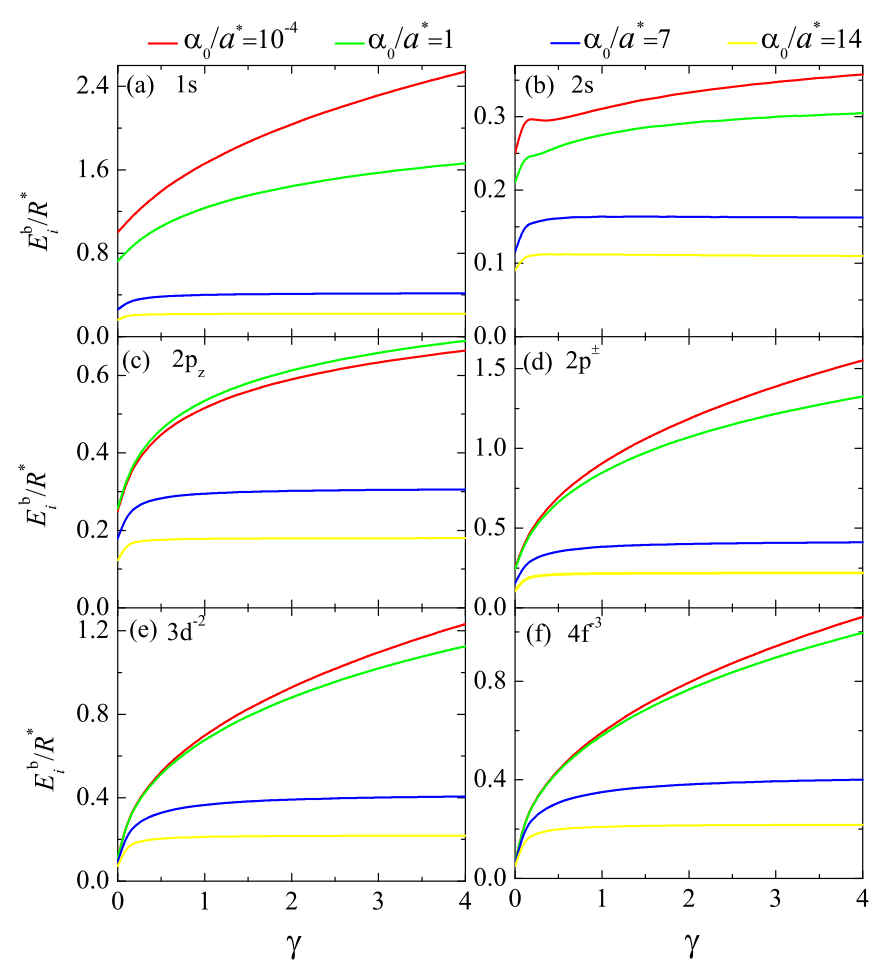

FIG. 5. The dependence of the binding energy for few lowest donor states on the magnetic field parameter $\gamma$ for different $\alpha_{0}$ as indicated.

when $\alpha_{0}$ changes from $a$ to $b, V(z, a)$ decreases in the region with small $z$ but increases in the region with large value of $z$, thus on the average $V(z, a)$ increases with increasing $\alpha_{0}$ and becomes largest at point $b$. After that the average $V(z, a)$ decreases with increasing $\alpha_{0}$ and becomes equivalent to the bare Coulomb potential at point $c$, and then it rapidly decreases with increasing $\alpha_{0}$. In sharp contrast, when $\alpha_{0}$ changes from $a$ to $d, V(\rho, a)$ decreases with increasing $\alpha_{0}$. In this way, the donor states with odd $z$ parity are more sensitive to $V(z, a)$ than to $V(\rho, a)$, whereas the other donor states behave in an opposite way. As a result, the width of the $2 p_{z}$ state exhibits a nonmonotonic dependence on $\alpha_{0}$ while that of the other states exhibits a monotonic dependence on $\alpha_{0}$ as shown in Fig. 3, leading to the novel behavior of the donor binding energy as observed in Fig. 2. Note that we found that the binding energy of the donor state with odd $z$ parity exhibits a nonmonotonic dependence on the laser-dressing parameter $\alpha_{0}$ in laser-driven semiconductor systems (see Appendix).

\section{B. Magnetic field effect on the donor binding energy}

Figure 5 shows the dependence of the binding energy for the few lowest donor states as a function of the magnetic field $\gamma$ for different $\alpha_{0}$. As $\alpha_{0}$ increases the binding energy for the $1 s, 2 s, 2 p^{ \pm}, 3 d^{-2}$, and $4 f^{-3}$ states becomes smaller than the static one and becomes more and more remarkable with increase of $\alpha_{0}$. In contrast, the binding energy for the $2 p_{z}$ state becomes larger than the static one with $\alpha_{0}$ up to the value of $\alpha_{0} / a^{*}=1$, and then it begins to decrease with increasing $\alpha_{0}$ and becomes considerable smaller than the static one when $\alpha_{0} / a^{*} \geqslant 7$. Moreover, the binding energy for 

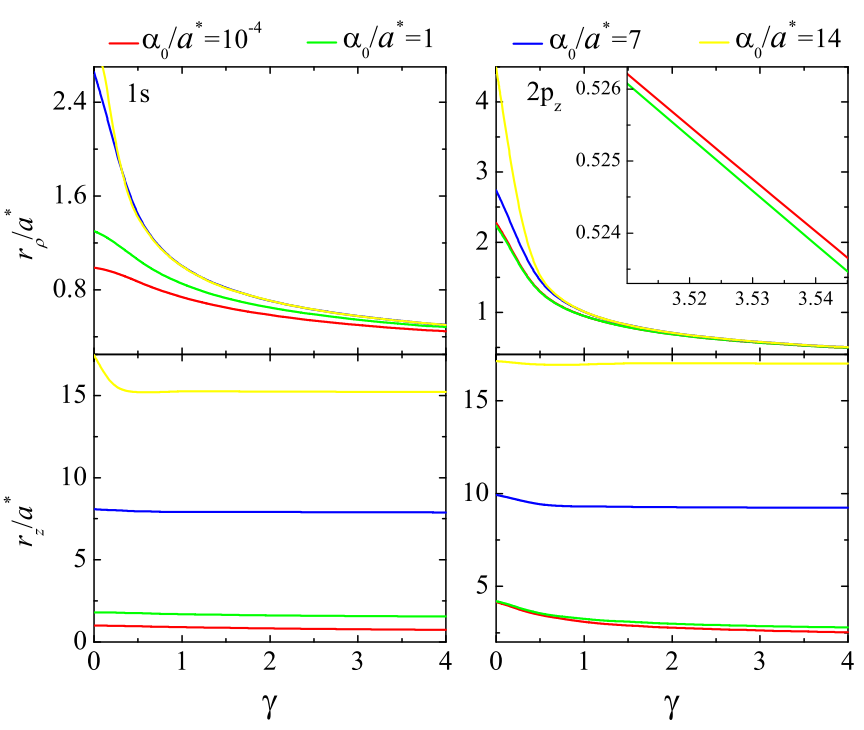

FIG. 6. The effective width of the wave functions for the $1 s$ and $2 p_{z}$ states in the $x-y$ plane (top figure) and in the $z$ direction (bottom figure) as a function of the magnetic field for different $\alpha_{0}$ as indicated. Inset: The region with large $\gamma$ is enlarged.

all donor states increases with increasing $\gamma$ when $\alpha_{0} / a^{*} \leqslant 1$, while it becomes insensitive to the magnetic field in the region with $\gamma>1$ when $\alpha_{0} / a^{*} \geqslant 7$.

The above behavior of the binding energy can be understood from Fig. 6, where the effective widths $r_{\rho}$ and $r_{z}$ for the $1 s$ and $2 p_{z}$ states are plotted as a function of the magnetic field for different $\alpha_{0}$. Here we only plot the width for two typical states because the magnetic field effect on the binding energy for the other states is qualitative similar to that of the $1 s$ state as shown in Fig. 5. Note that, when $\alpha_{0} / a^{*}=10^{-4}$, the widths $r_{\rho}$ and $r_{z}$ remain the static ones related to the absence of an ITLF.

Figure 6 shows that both $r_{\rho}$ and $r_{z}$ for the $1 s$ state become larger than the static ones with increasing $\alpha_{0}$, indicating that the electron wave function is more spread out, which leads to a smaller binding energy when compared with the static one as shown in Fig. 5(a). A similar dependence of $r_{z}$ for the $2 p_{z}$ state on $\alpha_{0}$ is also noticed. However, $r_{\rho}$ for the $2 p_{z}$ state becomes smaller than the static one with $\alpha_{0}$ up to the value of $\alpha_{0} / a^{*}=1$, after that it increases rapidly with increasing $\alpha_{0}$ and becomes larger than the static one when $\alpha_{0} / a^{*} \geqslant 7$, suggesting that the impurity-electron localization of the $2 p_{z}$ state in the $x-y$ plane first increases, but after a critical value of $\alpha_{0}$, it decreases rapidly with $\alpha_{0}$, resulting in a nonmonotonic behavior of the binding energy for the $2 p_{z}$ state as shown in Fig. 5(c). This is a consequence of the fact that the impurityelectron localization of the $2 p_{z}$ state in the $x-y$ plane plays a crucial role in determining its binding energy, since $r_{\rho}$ is much smaller than $r_{z}$ as shown in Fig. 6.

Moreover, $r_{z}$ for the $1 s$ and $2 p_{z}$ states almost does not depend on the magnetic field in the region with $\gamma \geqslant 1$ while $r_{\rho}$ for these two states decreases with increasing $\gamma$. Note that the LdCP is only affected by the ITLFs. The LdCP is remarkably weakened with respect to the bare Coulomb potential when $\alpha_{0} / a^{*} \geqslant 7$, which is evident by the fact that $r_{z}$ becomes significant larger than the static width as shown
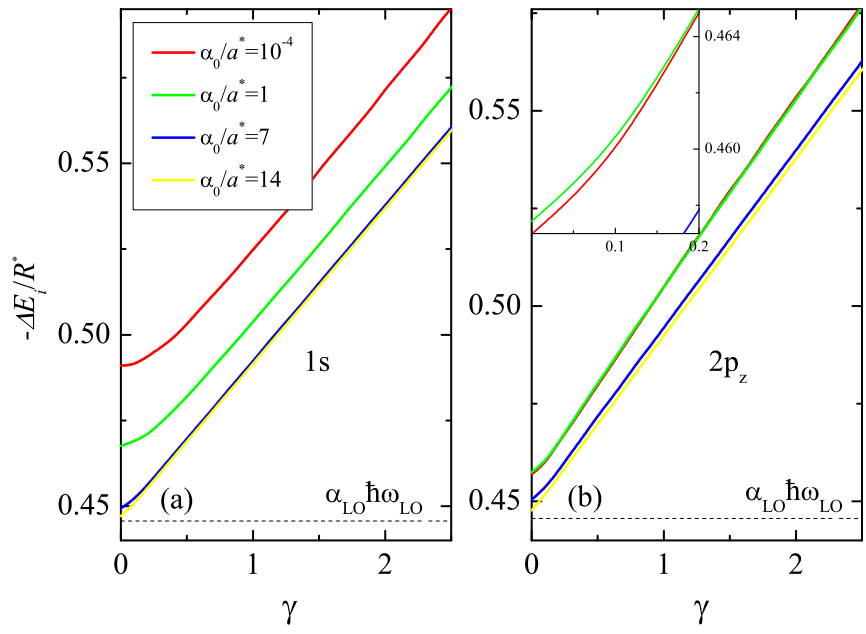

FIG. 7. Shifts of the energy levels due to the electron-LO-phonon interaction vs the magnetic field for a donor in GaAs for different $\alpha_{0}$ as indicated. $\alpha_{\mathrm{LO}} \hbar \omega_{\mathrm{LO}}$ indicates the polaron shift of a free electron in the field-free case. In (b), the region with small $\gamma$ is enlarged.

in Fig. 6, resulting in a reduction of the Coulomb force such that it becomes much smaller than the magnetic force. As a result, the bound states are lying just below the relevant LLs of a free electron when $\alpha_{0} / a^{*} \geqslant 7$, that is, the donor energy levels and the relevant LLs have almost the same dependence on the magnetic field, leading to a magnetic field independent donor binding energy in the region with $\gamma \geqslant 1$ as shown in Fig. 5.

\section{C. laser-modulated magnetopolaron correction}

In order to investigate how the ITLFs modulate the MPC in low magnetic field (i.e., below polaron resonant region), the MPC is calculated by using Eq. (17) for the $2 p^{+}$state and Eq. (16) for the other states. For simplicity, we only consider the case of $f=1 \mathrm{THz}$ with single-photon process as a representative example. In Fig. 7 we depict the MPC to the $1 s$ and $2 p_{z}$ states for different $\alpha_{0}$, where the polaron shift of a free electron in the field-free case, i.e., $\alpha_{\mathrm{LO}} \hbar \omega_{\mathrm{LO}}$, is indicated for reference. Here we do not plot the MPC for the other states since the behavior of the binding energy and the width are similar to that of the $1 s$ state. Note that when $\alpha_{0} / a^{*}=10^{-4}$, the MPC remains the static one related to the absence of an ITLF.

In Fig. 7 as expected the MPC increases with increasing $\gamma$ due to an additional lateral electronic confinement induced by the magnetic field, leading to $\left|\Delta E_{i}\right| \geqslant \alpha_{\mathrm{LO}} \hbar \omega_{\mathrm{LO}}$. Moreover, the MPC to the $1 s$ state can be effectively modulated by the ITLFs to be redshifted with respect to the static MPC, whereas that of the $2 p_{z}$ state can be adjusted to be not only redshifted but also blueshifted, which are physically consistent with the behavior of their width as shown in Fig. 6. However, the amount by which the MPCs are red- and blueshifted are much smaller than that of the binding energy, which is a result of the fact that the LdOP for the e-p coupling decreases with increasing $\alpha_{0}$, as reflected in Eq. (12).

Figure 8 shows the explicit dependence of the MPC for the $2 p^{+}$state with and without single-photon process on the 


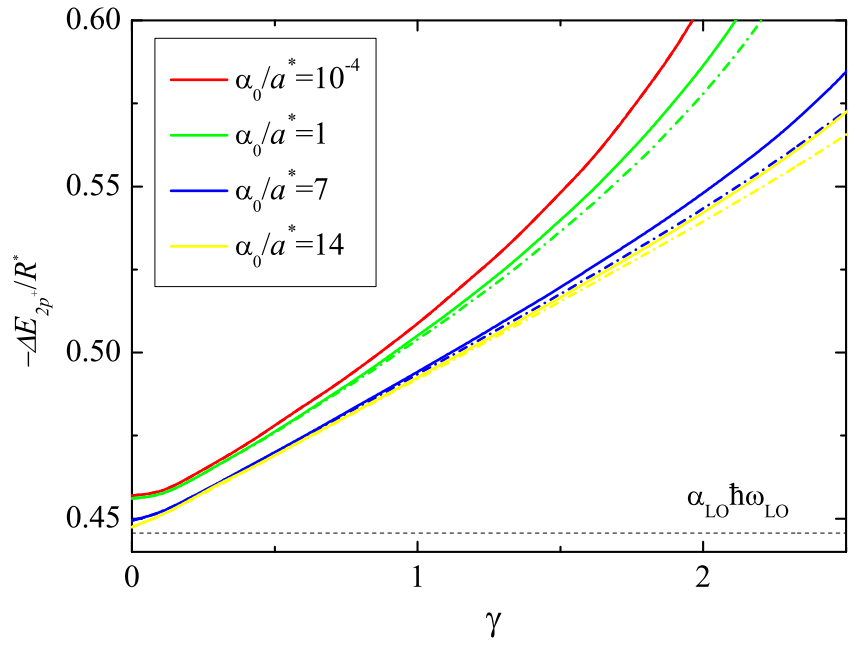

FIG. 8. Shift of energy levels of the $2 p^{+}$state with (solid curves) and without (short dashed-dotted curves) single-photon process vs the magnetic field for a donor in GaAs at fixed radiation frequency $f=1 \mathrm{THz}$ for different $\alpha_{0}$ as indicated. Here $\alpha_{\mathrm{LO}} \hbar \omega_{\mathrm{LO}}$ is shown to indicate the polaron shift of a free electron in the field-free case.

magnetic field at fixed $f=1 \mathrm{THz}$ for several values of $\alpha_{0}$. From this, it is clear that the MPC for the $2 p^{+}$state without single-photon process exhibits a behavior similar to that of the $1 s$ state due to the similar behavior of the impurity-electron localization, which rapidly increases with increasing $\gamma$ when $\gamma>1.5$ since the $2 p^{+}$state is moving close to resonance [3]. The MPC with single-photon processes is equivalent to that without single-photon process when $\alpha_{0} / a^{*}=10^{-4}$, indicating that the effect of the photon process on the MPC can be neglected. On the contrary, when $\alpha_{0} / a^{*} \geqslant 1$, the MPC with single-photon process becomes considerably larger than that without single-photon process and becomes more and more significant with increasing $\gamma$, which is a consequence of the fact that a new scattering channel has been opened up for the MPC by considering the effect of the single-photon process and the donor states become more and more localized with increasing $\gamma$. This indicates that the effect of the photon process on the MPC can be detected experimentally.

\section{D. laser-modulated magnetopolaron effect}

Iteratively solving Eq. (17), we find the laser-modulated MPE accompanied by the emission and absorption of a single photon. Our numerical results for the $1 s \rightarrow$ $2 s, 2 p^{ \pm}, 2 p_{z}, 3 d^{-2}$, and $4 f^{-3}$ transition energies including the effect of polaron, single-photon process, and band nonparabolicity are shown in Figs. 9 and 10 by the solid curves as a function of the magnetic field $\gamma$. We fix $f=1 \mathrm{THz}$ for several different values of $\alpha_{0}$, in which the MPE is accompanied by elastic scattering (ם) and absorption ( $\mathbf{\Lambda}$ ) and emission $(\star)$ of a single photon. Different branches of the $1 s \rightarrow 2 p^{+}$transition are marked with different colors in the same scattering channel. As a reference we have also plotted the transition energies without any correction indicated by thin dashed curves. For comparison, moreover, the results including the effect of polaron and band nonparabolicity (short dashed-dotted curves) and including the effect of single-photon process and polaron (dotted curves) are also depicted in these figures, respectively.

Figure 9 shows that, without considering the influence of the single-photon process, near resonance only five branches of the $1 s \rightarrow 2 p^{+}$transition are observed which are a consequence of the liftings of the $E_{2 p^{+}}^{(0)}$ and $E_{i}^{(0)}+$ $\hbar \omega_{\mathrm{LO}}\left(i=1 s, 2 p^{-}, 3 d^{-2}, 4 f^{-3}, 2 p_{z}\right)$ degeneracies. In contrast, ten additional branches of the $1 s \rightarrow 2 p^{+}$transition are observed in the resonant region when the effect of the single-photon process have been taken into account, resulting from the liftings of the $E_{2 p^{+}}^{(0)}$ and $E_{i}^{(0)}+\hbar \omega_{\mathrm{LO}} \pm \hbar \omega(i=$ $1 s, 2 p^{-}, 3 d^{-2}, 4 f^{-3}, 2 p_{z}$ ) degeneracies which correspond to the MPE accompanied by the absorption and emission of a single photon. This is similar to the results previously obtained in the Faraday configuration, except for the cyclotron motion [28]. Most importantly, the additional branches of the $1 s \rightarrow 2 p^{+}$transition can be tuned to be far away from the reststrahlen band by changing the radiation frequency and, therefore, leading to the observation of a MPE accompanied by the absorption and emission of a single photon in low magnetic field.

By comparing Fig. 9(b) with Fig. 10, we can see that a remarkable characteristic anticrossing gap of the MPE accompanied by the absorption and emission of a single photon is observed when $\alpha_{0} / a^{*}=1$, which is comparable to that without the single-photon process and thus changes the donor energy levels appreciably in the resonant region. However, when the radiation level is intense enough, i.e., when $\alpha_{0} / a^{*} \geqslant$ 7 , the characteristic anticrossing gap become unclear, which is a consequence of the fact that the donor energy levels approach each other due to the weakening of the LdCP in this case and the LdOP for the e-p coupling decreases with increasing $\alpha_{0}$. This implies that the magnetic and ITLFs can be properly chosen to generate the desired MPE accompanied by the absorption and emission of a single photon in this laser-driven semiconductor system, which in turn provides a new direct measure of the e-p interaction in low magnetic field.

Figures 9 and 10 also show that the $1 s \rightarrow 2 s, 2 p^{ \pm}$, $2 p_{z}, 3 d^{-2}$, and $4 f^{-3}$ transition energies increase with increasing $\gamma$ when $\alpha_{0} / a^{*} \leqslant 10^{-4}$, which results from the additional lateral confinement induced by the magnetic field. However, the $1 s \rightarrow 2 p^{-}, 3 d^{-2}$, and $4 f^{-3}$ transition energies become a slightly decreasing function of $\gamma$ when $\alpha_{0} / a^{*}=1$ and become a rapid decreasing function of $\gamma$ with further increase of $\alpha_{0}$. This is due to the fact that the dependence of the energy levels of the $2 p^{-}, 3 d^{-2}$, and $4 f^{-3}$ states on the magnetic field is slightly weaker than that of the $1 s$ state and become more and more significant with increasing $\alpha_{0}$. On the contrary, the $1 s \rightarrow 2 s$ and $2 p_{z}$ transition energies become insensitive to the magnetic field in the region with $\gamma>1$ when $\alpha_{0} / a^{*}=$ 7 because these donor energy levels have almost the same dependence on magnetic field. In addition, the effect of the band nonparabolicity on the donor energy levels become more and more important with increasing $\alpha_{0}$.

To demonstrate feasibility of experimental observations of the laser-modulated MPE, we calculate the oscillator strength (OS) and the absorption cross section (ACS) for the transitions shown in Figs. 9 and 10. This is because the OS and ACS 

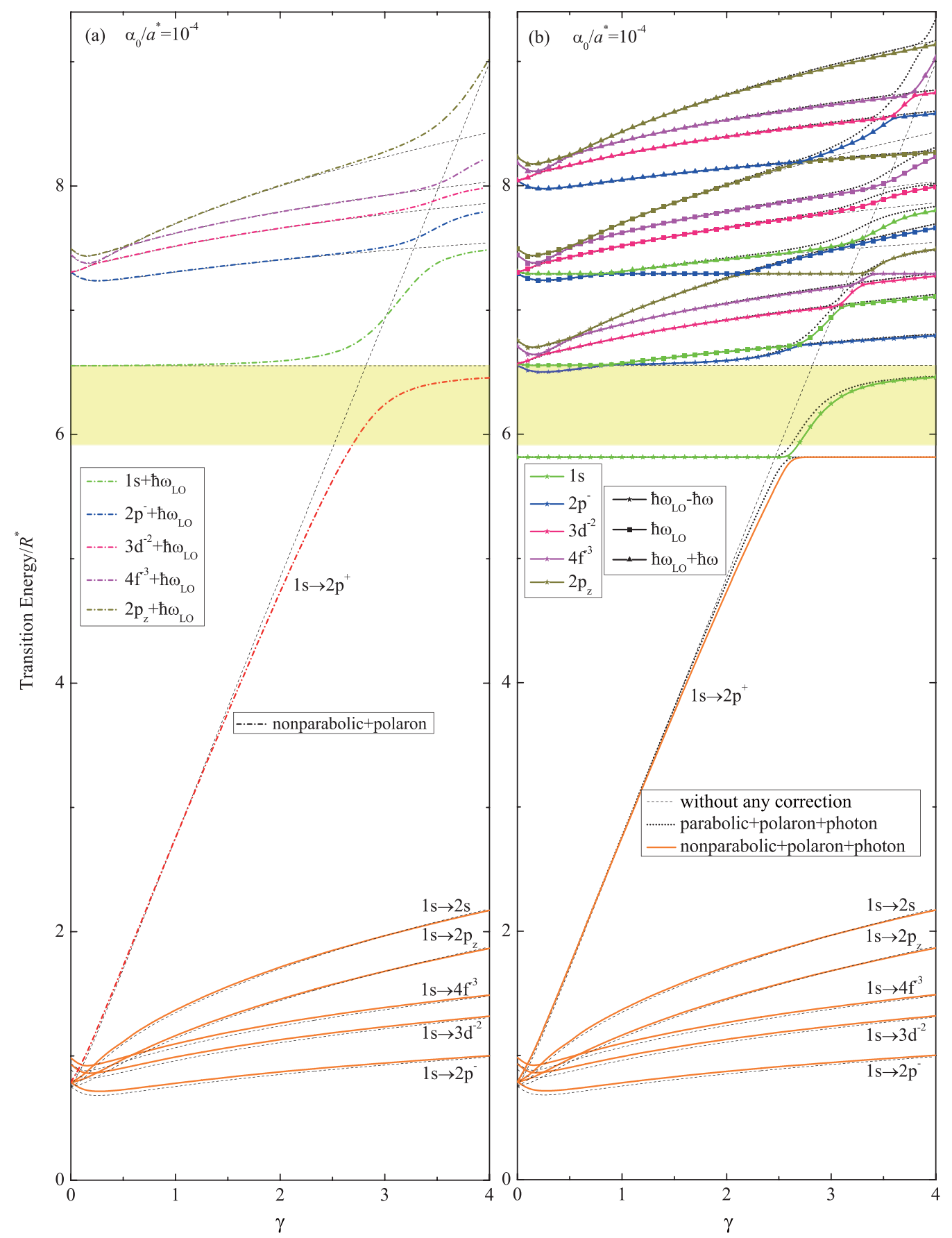

FIG. 9. Transition energy as a function of the magnetic field for several major donor transitions at fixed $f=1 \mathrm{THz}$ and $\alpha_{0} / a^{*}=10^{-4}$ when the MPE (a) without and (b) with the effect of the single-photon process. For comparison, we consider the following cases: (1) without any correction (thin dashed curves); (2) with the polaron effect and band nonparabolicity; (3) including the polaron effect and single-photon process (dotted curves); and (4) including the polaron effect, single-photon process, and band nonparabolicity (solid curves), in which the MPE is accompanied by the elastic-photon scattering ( $\mathbf{\square})$ and the emission ( $\star$ ) and absorption ( $\mathbf{\Delta})$ of single photon. In (a) and (b) the yellow shaded rectangle denotes the reststrahlen band.

can be detected in experiments [43]. The OS $f_{i j}$ and ACS $\sigma$ for a transition from energy level $i$ to energy level $j$ are, respectively, given by $[39,43,44]$

$$
f_{i j}=\frac{2 m^{*}\left(E_{j}^{(0)}-E_{i}^{(0)}\right)}{\hbar^{2}}|\langle j|\vec{e} \cdot \vec{R}| i\rangle|^{2}
$$

and

$$
\sigma=\frac{4 \pi^{2} \alpha\left(E_{j}^{(0)}-E_{i}^{(0)}\right)}{\hbar}|\langle j|\vec{e} \cdot \vec{R}| i\rangle|^{2} \delta\left(E_{j}^{(0)}-E_{i}^{(0)}-\hbar \Omega\right) .
$$

Here $\alpha$ is the fine structure constant, and $\vec{e}$ and $\hbar \Omega$ are the unit polarization vector of the incident optical field and its photon energy, respectively. In the presence of broadening 


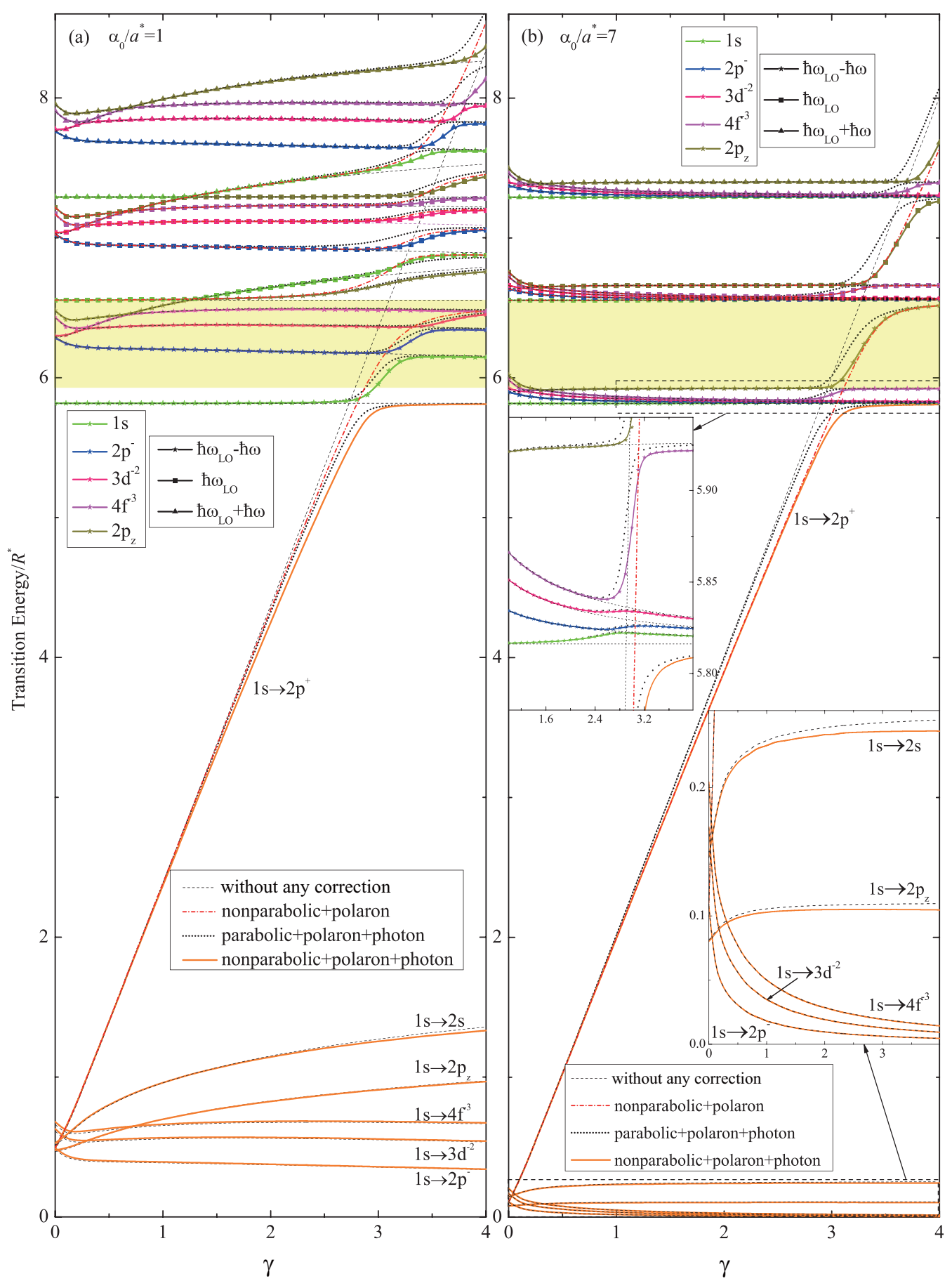

FIG. 10. The same as Fig. 9 for (a) $\alpha_{0} / a^{*}=1$ and (b) $\alpha_{0} / a^{*}=7$. The marked areas are enlarged in the insets.

of the shallow-donor states, the $\delta$ function in ACS can be replaced by a narrow Lorentzian by means of

$$
\delta\left(E_{j}^{(0)}-E_{i}^{(0)}-\hbar \Omega\right)=\frac{\hbar \Gamma / \pi}{\left(E_{j}^{(0)}-E_{i}^{(0)}-\hbar \Omega\right)^{2}+(\hbar \Gamma)^{2}},
$$

where $\Gamma$ is the donor linewidth, which we takes as $0.1 R^{*}$ in line with literature [45]. The results are shown in Figs. 11 and 12, respectively. Here we do not plot the OS and the ACS for the other transitions since the behaviors of these transitions are similar to that of $1 s \rightarrow 2 p^{ \pm}$and $2 p_{z}$ transitions. From
Figs. 11 and 12 it is clear that both OS and ACS for the $1 s \rightarrow$ $2 p^{+}$and $2 p_{z}$ transitions increase with increasing $\alpha_{0}$, indicating that these two transitions can be enhanced by the ITLF with respect to the static one. In sharp contrast, both OS and ACS for the $1 s \rightarrow 2 p^{-}$transition decrease with increasing $\alpha_{0}$, however, it still can be clearly observed with an appropriate choice of the ITLF intensity. Note that the OSs discussed here are larger than unity, which does not violate the sum rule for OS since the transitions studied represent only a small fraction of all possible transitions in the electronic spectrum. Similar relative OS has been reported in the literature [46]. 


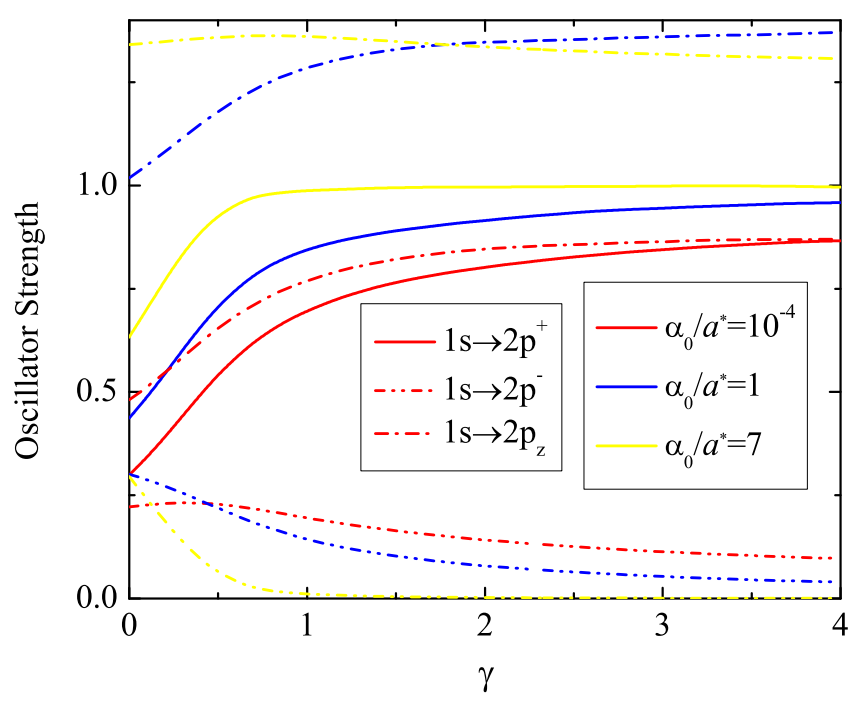

FIG. 11. The OS for the $1 s \rightarrow 2 p^{ \pm}$and $2 p_{z}$ transitions as a function of the magnetic field for different $\alpha_{0}$ as indicated.

The ACS has the same order of magnitude as previous experimental results in Ref. [43]. Most importantly, the static transitions shown in this paper have been already observed experimentally in the absence of the ITLF [3]. Moreover, the current generation of FELs can provide intense laser fields in the THz bandwidth, which makes our theoretical findings testable. This analysis shows that the laser-modulated MPE observed in Figs. 9 and 10 can be realized in experiments by using THz FEL radiation.

To further confirm feasibility of experimental observations of the laser-modulated MPE, we also investigate how the ITLF modulate the matrix elements of the LdOP for e-p interaction and, therefore modulate the MPE in such laser-driven semiconductor systems. The matrix elements of the LdOP for e-p
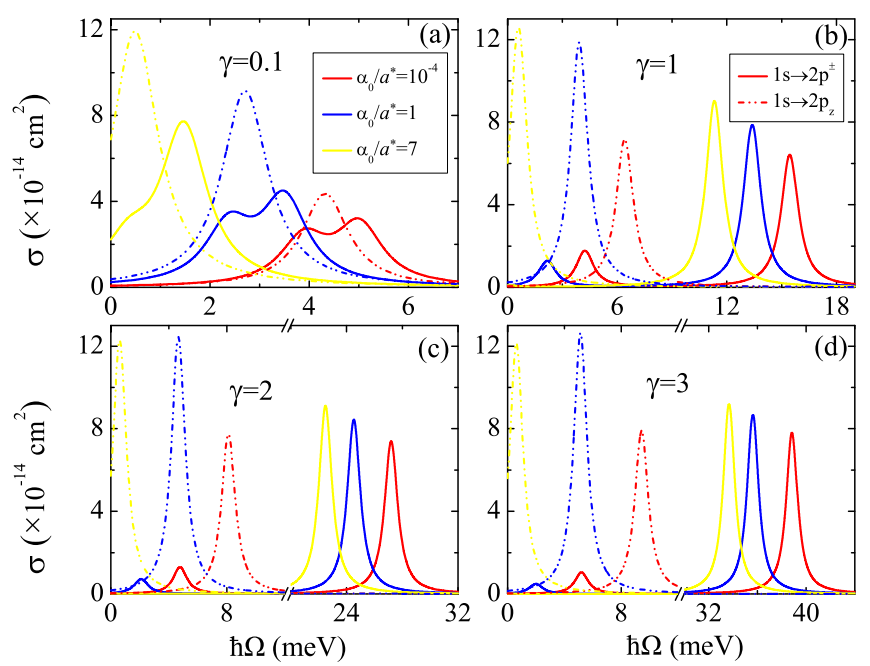

FIG. 12. The ACS for the $1 s \rightarrow 2 p^{ \pm}$and $2 p_{z}$ transitions as a function of the incident photon energy $\hbar \Omega$ for different $\gamma$ and $\alpha_{0}$ as indicated.

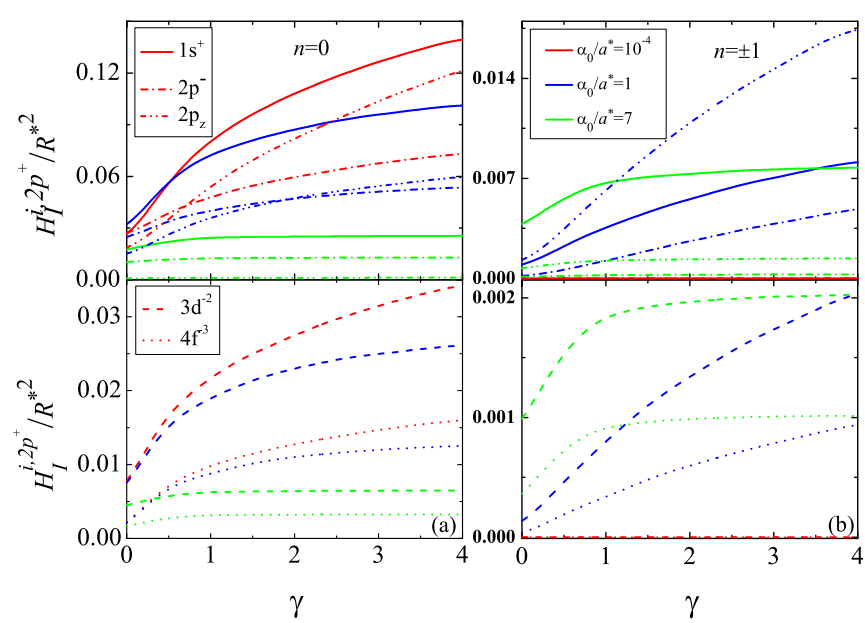

FIG. 13. The values of the LdOP transition matrix elements as a function of the magnetic field for different $\alpha_{0}$ when the LdOP transition matrix elements (a) without and (b) with the effect of the single-photon process.

interaction is given by

$$
H_{I}^{i, j}=\sum_{n, \vec{Q}}\left|\left\langle i ; \vec{Q}\left|H_{e p}(\vec{R})\right| j ; \overrightarrow{0}\right\rangle\right|^{2} J_{n}^{2}\left(q_{z} \alpha_{0}\right),
$$

which represents the transition probability between the $|i \vec{Q}\rangle$ and $|j ; \overrightarrow{0}\rangle$ states. The value of $H_{I}^{i, j}$ is a measure of how strong the LdOP for e-p interaction couples these states with each other. The results of $H_{I}^{i, j}$ are shown in Fig. 13. Here we only consider $H_{I}^{i, 2 p^{+}}\left(i=1 s, 2 p^{-}, 2 p_{z}, 3 d^{-2}, 4 f^{-3}\right)$ that is relevant to Figs. 9 and 10. Figure 13 shows that, without considering the effect of the single-photon process, $H_{I}^{i, 2 p^{+}}$ $\left(i=1 s, 2 p^{-}, 2 p_{z}, 3 d^{-2}, 4 f^{-3}\right)$ decreases with increasing $\alpha_{0}$, however, which is still strong enough to be observed clearly. On the contrary, with including the effect of the singlephoton process, $H_{I}^{i, 2 p^{+}}\left(i=1 s, 3 d^{-2}, 4 f^{-3}\right)$ increases with increasing $\alpha_{0}$, while $H_{I}^{i, 2 p^{+}}\left(i=2 p^{-}, 2 p_{z}\right)$ first increases and then decreases with increasing $\alpha_{0}$. This indicates that $H_{I}^{i, 2 p^{+}}$ $\left(i=1 s, 2 p^{-}, 2 p_{z}, 3 d^{-2}, 4 f^{-3}\right.$ ) can be effectively modulated by the ITLF, leading to a laser-modulated MPE since the intensity of the MPE is directly proportional to $H_{I}^{i, 2 p^{+}}$( $i=$ $1 s, 2 p^{-}, 2 p_{z}, 3 d^{-2}, 4 f^{-3}$ ) as reflected in Eq. (14). Most importantly, the value of $H_{I}^{i, 2 p^{+}}\left(i=1 s, 2 p^{-}, 2 p_{z}, 3 d^{-2}, 4 f^{-3}\right)$ with single-photon process is about $12 \%$ of that without single-photon process for the same ITLF intensity when $\alpha_{0} / a^{*} \geqslant 1$, which is strong enough to be detected in magnetooptical experiments. Note that the static MPE on shallowdonor states in semiconductors has been already observed experimentally in the absence of the ITLF [3]. The above results indicate that the laser-modulated MPE can be verified experimentally with present-day techniques.

\section{E. Dynamical magnetopolaron effect}

Up to now we find that not only the binding energy but also the MPE accompanied by the absorption and emission of a single photon can be tuned by the ITLFs. In particular, 


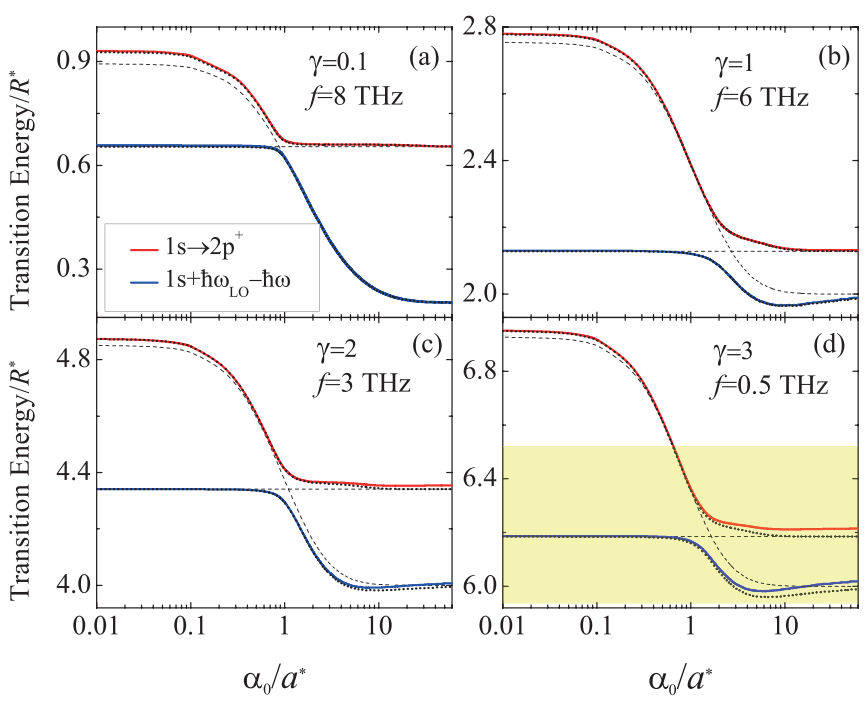

FIG. 14. The $1 s \rightarrow 2 p^{+}$transition energy as a function of $\alpha_{0}$ for several values of $\gamma$ and $f$ when the MPE accompanied by the emission of single photon. For comparison, we consider the following cases: (1) without any correction (thin dashed curves); (2) with the polaron effect and single-photon process (dotted curves); and (3) with the polaron effect, single-photon process, and band nonparabolicity (solid curves). In (d) the yellow shaded rectangle denotes the reststrahlen band.

a significant characteristic anticrossing gap is observed for the branch $E_{1 s}^{(0)}+\hbar \omega_{\mathrm{LO}}-\hbar \omega$ of the MPE and its resonant region can be adjusted by changing the radiation frequency. In this way, the $1 s \rightarrow 2 p^{+}$transition accompanied by the emission of a single photon can be tuned by the ITLFs through the resonance energy with the LO phonon. The results for the $1 s \rightarrow 2 p^{+}$transition energy including the polaron effect, single-photon process, and band nonparabolicity are plotted by the solid curves in Fig. 14 as a function of $\alpha_{0}$, where the transition energies without any correction are indicated by the thin dashed curves for reference. As a comparison we have also plotted the results including the polaron effect and single-photon process (dotted curves) in this figure.

From Fig. 14 it is clear that near resonance two transitions are observed which are a consequence of the liftings of the $E_{2 p^{+}}^{(0)}$ and $E_{1 s}^{(0)}+\hbar \omega_{\mathrm{LO}}-\hbar \omega$ degeneracy, corresponding to a resonant effect accompanied by the emission of a single photon, which is similar to the well-known MPE observed above. Since this effect occurs by changing the ITLFs which is different from the MPE observed by changing the magnetic field, we thus call it the dynamical magnetopolaron effect (DMPE). A considerable characteristic anticrossing gap for the DMPE is observed in low magnetic field, which can be adjusted to be far away from the reststrahlen band with an appropriate choice of the radiation frequency. This in turn also provides a new way to measure the strength of the e-p interaction in low magnetic field.

To demonstrate feasibility of experimental observations of the DMPE, we calculate the OS and $H_{I}^{1 s, 2 p^{+}}$with singlephoton process as a function of $\alpha_{0}$ for different magnetic fields. The results are shown in Figs. 15 and 16, respectively. From Fig. 15 it is apparent that the OS is an increasing

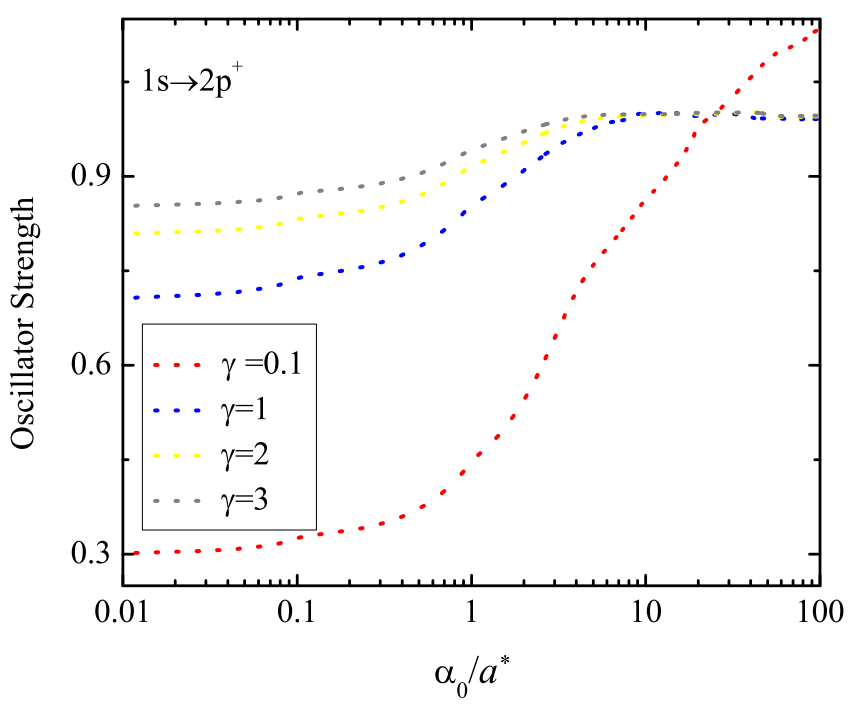

FIG. 15. The OS for the $1 s \rightarrow 2 p^{+}$transition as a function of $\alpha_{0}$ for different $\gamma$ as indicated. Here $\gamma=0.1,1,2,3$ correspond to Figs. 14(a)-14(d), respectively.

function of $\alpha_{0}$, resulting in a remarkable $1 s \rightarrow 2 p^{+}$transition with respect to the static one, which indicates that the $1 \mathrm{~s} \rightarrow$ $2 p^{+}$transition can be observed experimentally. Figure 16 shows that, as $\alpha_{0}$ increases, $H_{I}^{1 s, 2 p^{+}}$with single-photon process increases rapidly at first, after it reaches its maximum, $H_{I}^{1 s, 2 p^{+}}$rapidly decreases with increasing $\alpha_{0}$. This implies that the DMPE can be tuned by the ITLF since the intensity of DMPE is directly proportional to $H_{I}^{1 s, 2 p^{+}}$with a singlephoton process. By comparing Fig. 13 with 16, one can see that the intensity of peak shown in Fig. 16 is about $12 \%$ of those without single-photon process for the same magnetic field as shown in Fig. 13(a), which is also strong enough to be detected in magneto-optical experiments, leading to a

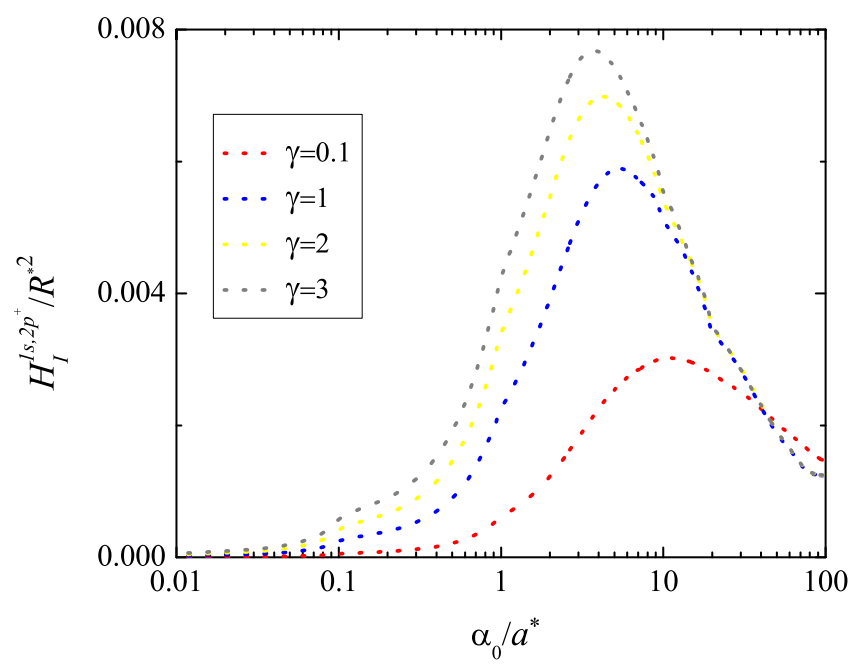

FIG. 16. The values of the LdOP transition matrix elements for the $1 s \rightarrow 2 p^{+}$transition with single-photon process as a function of $\alpha_{0}$ for different $\gamma$ as indicated. Here $\gamma=0.1,1,2,3$ correspond to Figs. 14(a)-14(d), respectively. 
observable DMPE on shallow-donor states in the laser-driven semiconductor systems.

\section{CONCLUSIONS}

In this study, within the Voigt configuration, we have studied the characteristics of shallow-donor states in GaAs semiconductors subjected simultaneously to a static magnetic and ITLFs. By using a nonperturbative approach in combination with a variational method, the ITLF effects on the donor binding energy are investigated in which the ITLF effects are exactly taken into account through the LdCP for electronimpurity interaction and the LdOP for e-p interaction. We used the TdPT to theoretically study the laser-modulated MPE accompanied by the absorption and emission of a single photon and the DMPE on donor states in this laser-driven semiconductor. In conjunction with optical measurements, the intradonor transition energy including the polaron effect, single-photon process, and band nonparabolicity was calculated and discussed. The main conclusions obtained from the present study are summarized as follows.

We found that the interaction strength of the LdCP in the $z$ direction can be not only enhanced but also weakened with increasing $\alpha_{0}$, while that in the $x-y$ plane is monotonically weakened, so that the electron localization of the $2 p_{z}$ state can be tuned by the ITLFs and increased or decreased with respect to the static electron localization while that of the other states decreases with increasing $\alpha_{0}$. For these reasons, the binding energy of the $2 p_{z}$ state can be larger or smaller than the static binding energy while that of the other states becomes smaller than the static one. Moreover, when $\alpha_{0} / a^{*} \leqslant 1$, the binding energy of the donor states is an increasing function of the magnetic field, while it becomes insensitive to the magnetic field for $\gamma \geqslant 1$ when $\alpha_{0} / a^{*} \geqslant 7$.

In the polaron nonresonant region, as $\alpha_{0}$ increases, the MPC for the $2 p_{z}$ state can either increase or decrease with respect to the static MPC while that for the other states only decreases with increasing $\alpha_{0}$, which is physically consistent with the variation of the width for the donor states with $\alpha_{0}$. By considering the effect of single-photon process, the MPC for the $2 p^{+}$state is larger than that without a single-photon process, which results from the fact that a new scattering channel has been opened up for the MPC in this case.

In the resonant region, the laser-modulated MPE accompanied by the absorption and emission of a single photon is observed, which can be tuned to be far away from the reststrahlen band by changing the radiation frequency. Ten additional branches of the $1 s \rightarrow 2 p^{+}$transition are observed and its characteristic anticrossing gap can be adjusted to be significant by varying the ITLFs, which indicates that such laser-modulated MPE can be detected experimentally. Interestingly, a novel DMPE with a remarkable characteristic anticrossing gap is clearly observed with an appropriate choice of the radiation frequency. These novel effects provide a new direct measure of the e-p interaction in laser-driven semiconductor systems in low magnetic field.

We have demonstrated that the intriguing laser-modulated MPE accompanied by the emission and absorption of a single photon and the novel DMPE on shallow-donor states in GaAs semiconductors can be measured in the presence of the ITLFs.

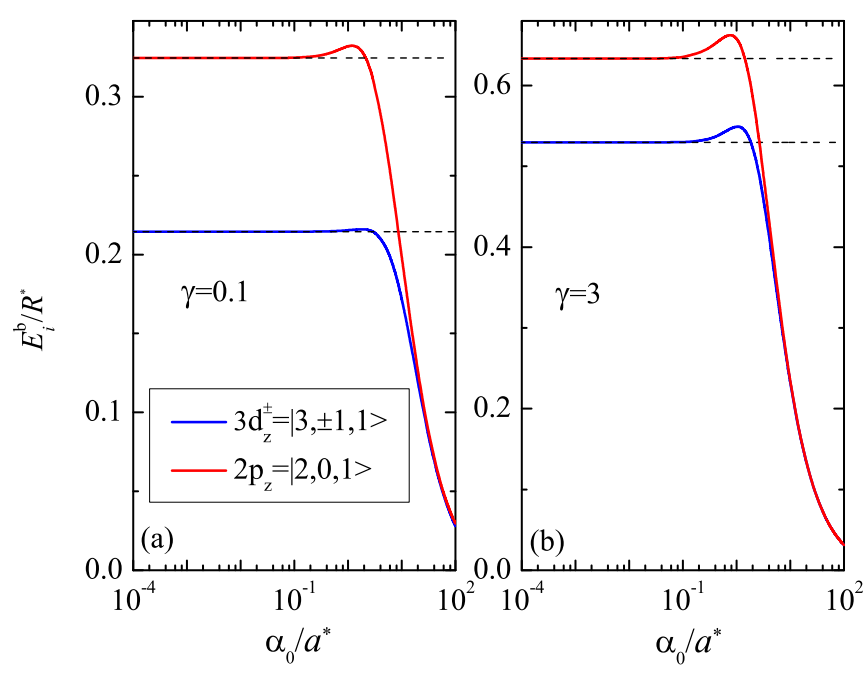

FIG. 17. The dependence of the binding energy of the states with odd $z$ parity, i.e., $3 d_{z}^{ \pm}=|3, \pm 1,1\rangle$ and $2 p_{z}=|2,0,1\rangle$, as a function of $\alpha_{0}$ at fixed (a) $\gamma=0.1$ and (b) $\gamma=3$. Here the horizontal dashed lines represent the static binding energy in the absence of the ITLFs.

Currently, THz FELs have been used to investigate various materials including doped semiconductors, which makes our results experimentally testable. We, therefore, hope that our theoretical predictions can be verified experimentally in the near future.

In our previous Faraday configuration [28], the interaction strength of the LdCP cannot only be enhanced but also weakened by varying the magnetic field and the ITLF, leading to an appreciable effect of cyclotron motion on the electronic property of shallow-impurity states. In this way, the binding energy and the MPC for all shallow-impurity states can be increased or decreased with respect to the static one. Meanwhile, all binding energies and all transition energies increase with increasing magnetic field. In sharp contrast, in the Voigt configuration, the interaction strength of the LdCP is only affected by the ITLF, so that the effect of cyclotron motion cannot be observed. More interestingly, the interaction strength of the LdCP in the $x-y$ plane is only weakened by the ITLF, so that the binding energy and the MPC for all shallowimpurity states decrease with increasing $\alpha_{0}$ except for the states with odd $z$ parity. Moreover, when $\alpha_{0}$ is strong enough, all binding energies become insensitive to the magnetic field. Meanwhile, the $1 s \rightarrow 2 p^{-}, 3 d^{-2}$, and $4 f^{-3}$ transition energies become a rapid decreasing function of magnetic field, while the $1 s \rightarrow 2 s$ and $2 p_{z}$ transition energies become insensitive to the magnetic field. Finally, note that similar behaviors of the MPE have been observed in both configurations, but only the DMPE is reported in the present work.

\section{ACKNOWLEDGMENTS}

This work was supported by National Natural Science Foundation of China (Grants No. 11404214, No. 11455015, and No. 61504016) and the China Scholarship Council (CSC), and Science and Technology Research Foundation of Jiangxi Provincial Education Department (Grants No. GJJ161062 and No. GJJ180868). B.V.D. was supported by the Research 
Foundation - Flanders (FWO-Vl) through a postdoctoral fellowship.

\section{APPENDIX}

In this Appendix we investigate the dependence of the binding energy for the state with odd $z$ parity on the laserdressing parameter $\alpha_{0}$. The results for the $3 d_{z}^{ \pm}=|3, \pm 1,1\rangle$ and $2 p_{z}=|2,0,1\rangle$ states are shown in Fig. 17. From this it is apparent that the nonmonotonic behavior of the binding energy for the $3 d_{z}^{ \pm}$states in the ITLF is similar to that of the $2 p_{z}$ state. In other words, as $\alpha_{0}$ increases, the binding energy of the donor state with odd $z$ parity increases slightly at first, after reaching a maximum, the binding energy decreases rapidly. Thus, this nonmonotonic dependence of the binding energy on $\alpha_{0}$ is a characteristic property of the donor state with odd $z$ parity in laser-driven semiconductor systems.
[1] G. Lindemann, R. Lassnig, W. Seidenbusch, and E. Gornik, Phys. Rev. B 28, 4693 (1983); X. Wu, F. M. Peeters, and J. T. Devreese, ibid. 34, 8800 (1986); S. N. Klimin, V. M. Fomin, and J. T. Devreese, ibid. 77, 205311 (2008); K. Ju, C. Guo, and X. Pan, Chin. Phys. B 26, 007103 (2017); Q. Chen, W. Wang, and F. M. Peeters, J. Appl. Phys. 123, 214303 (2018).

[2] See, e.g., D. M. Larsen, Phys. Rev. B 30, 4595 (1984); U. Merkt, M. Horst, T. Evelauer, and J. P. Kotthaus, ibid. 34, 7234 (1986); X. Wu, F. M. Peeters, and J. T. Devreese, ibid. 34, 2621 (1986).

[3] J. M. Shi, F. M. Peeters, and J. T. Devreese, Phys. Rev. B 48, 5202 (1993); J.-P. Cheng, B. D. McCombe, J. M. Shi, F. M. Peeters, and J. T. Devreese, ibid. 48, 7910 (1993).

[4] J. M. Shi, F. M. Peeters, J. T. Devreese, Y. Imanaka, and N. Miura, Phys. Rev. B 52, 17205 (1995); M. Grynberg, S. Huant, G. Martinez, J. Kossut, T. Wojtowicz, G. Karczewski, J. M. Shi, F. M. Peeters, and J. T. Devreese, ibid. 54, 1467 (1996).

[5] R. A. Lewis, P. E. Simmonds, and Y.-J. Wang, Phys. Rev. B 72, 245207 (2005); A. Wysmołek, R. Stępniewski, M. Potemski, B. Chwalisz-Piętka, K. Pakuła, J. M. Baranowski, D. C. Look, S. S. Park, and K. Y. Lee, ibid. 74, 195205 (2006).

[6] B. A. Mason and S. Das Sarma, Phys. Rev. B 33, 8379 (1986); C. D. Hu and Y.-H. Chang, ibid. 40, 3878 (1989); D. L. Lin, R. Chen, and T. F. George, ibid. 43, 9328 (1991); R. Chen, K. K. Bajaj, J. P. Cheng, and B. D. McCombe, ibid. 51, 9325 (1995); F. A. P. Osório, M. Z. Maialle, and O. Hipólito, ibid. 57, 1644 (1998).

[7] J. M. Shi, F. M. Peeters, G. Q. Hai, and J. T. Devreese, Phys. Rev. B 44, 5692 (1991); J. M. Shi, F. M. Peeters, and J. T. Devreese, ibid. 51, 7714 (1995); F. M. Peeters, J. M. Shi, and J. T. Devreese, Phys. Scr. 55, 57 (1994).

[8] S. Hameau, Y. Guldner, O. Verzelen, R. Ferreira, G. Bastard, J. Zeman, A. Lemaître, and J. M. Gérard, Phys. Rev. Lett. 83, 4152 (1999); S. Hameau, J. N. Isaia, Y. Guldner, E. Deleporte, O. Verzelen, R. Ferreira, G. Bastard, J. Zeman, and J. M. Gérard, Phys. Rev. B 65, 085316 (2002); B. S. Kandemir and A. Çetin, ibid. 65, 054303 (2002); A. L. Vartanian, L. A. Vardanyan, and E. M. Kazaryan, Phys. Lett. A 360, 649 (2007).

[9] C. J. Joachain, N. J. Kylstra, and R. M. Potvliege, Atoms in Intense Laser Fields (Cambridge University Press, New York, 2012).

[10] For recent development, see, e.g., U. Yesilgul, Physica E 74, 34 (2015); E. Kasapoglu, S. Sakiroglu, I. Sökmen, R. L. Restrepo, M. E. Mora-Ramos, and C. A. Duque, Opt. Mater. 60, 318 (2016); E. C. Niculescu, Chem. Phys. 487, 16 (2017).

[11] M. Pont, N. R. Walet, M. Gavrila, and C. W. McCurdy, Phys.
Rev. Lett. 61, 939 (1988); M. Pont, N. R. Walet, and M. Gavrila, Phys. Rev. A 41, 477 (1990).

[12] F. M. S. Lima, O. A. C. Nunes, M. A. Amato, A. L. A. Fonseca, and E. F. da Silva, Jr., J. Appl. Phys. 103, 113112 (2008).

[13] Q. Su, J. H. Eberly, and J. Javanainen, Phys. Rev. Lett. 64, 862 (1990); M. Pont and M. Gavrila, ibid. 65, 2362 (1990); M. Dörr, R. M. Potvliege, D. Proulx, and R. Shakeshaft, Phys. Rev. A 43, 3729 (1991); R. Kundliya and M. Mohan, Phys. Lett. A 291, 22 (2001); M. Gavrila, J. Phys. B: At. Mol. Opt. Phys. 35, R147 (2002).

[14] F. M. S. Lima, M. A. Amato, L. S. F. Olavo, O. A. C. Nunes, A. L. A. Fonseca, and E. F. da Silva, Jr., Phys. Rev. B 75, 073201 (2007).

[15] R. B. Liu and B. F. Zhu, Phys. Rev. B 66, 033106 (2002).

[16] F. M. S. Lima, M. A. Amato, O. A. C. Nunes, A. L. A. Fonseca, and E. F. da. Silva, Jr., J. Phys. B: At. Mol. Opt. Phys. 42, 055601 (2009).

[17] A. L. A. Fonseca, M. A. Amato, and O. A. C. Nunes, Phys. Status Solidi B 186, K57 (1994); J. L. Nie, W. Xu, and L. B. Lin, Int. J. Mod. Phys. B 17, 2487 (2003).

[18] Q. Fanyao, A. L. A. Fonseca, and O. A. C. Nunes, Phys. Rev. B 54, 16405 (1996); J. Appl. Phys. 82, 1236 (1997); C. GonzálezSantander, T. Apostolova, and F. Domínguez-Adame, J. Phys.: Condens. Matter 25, 335802 (2013); D. G. Kilic, S. Sakiroglu, and I. Sokmen, Physica E 102, 50 (2018).

[19] H. S. Brandi, A. Latgé, and L. E. Oliveira, Phys. Status Solidi B 210, 671 (1998); Phys. Rev. B 64, 035323 (2001); 70, 153303 (2004).

[20] H. Sari, E. Kasapoglu, I. Sokmen, and N. Balkan, Semicond. Sci. Technol. 18, 470 (2003); H. Sari, F. Ungan, S. Sakiroglu, U. Yesilgul, and I. Sökmen, J. Phys. Chem. Solids 120, 279 (2018).

[21] W. Wang, L. Xu, B. Wu, S. Zhang, and X. Wei, Physica B 521, 122 (2017); X. Wei, W. Wang, L. Xu, S. Zhang, and R. Tan, Phys. Status Solidi B 255, 1800211 (2018).

[22] H. S. Brandi, A. Latgé, and L. E. Olieira, Solid State Commun. 107, 31 (1998); H. Sari, E. Kasapoglu, and I. Sökmen, Phys. Lett. A 311, 60 (2003); C. A. Duque, M. E. Mora-Ramos, E. Kasapoglu, H. Sari, and I. Sökmen, Eur. Phys. J. B 81, 441 (2011); C. A. Duque, E. Kasapoglu, S. Şakiroglu, H. Sari, and I. Sökmen, Appl. Surf. Sci. 257, 2313 (2011); E. C. Niculescu, Eur. Phys. J. B 85, 66 (2012); U. Yesilgul, H. Sari, F. Ungan, J. C. Martínez-Orozco, R. L. Restrepo, M. E. Mora-Ramos, C. A. Duque, and I. Sökmen, Chem. Phys. 485-486, 81 (2017); H. Sari, E. Kasapoglu, S. Sakiroglu, U. Yesilgul, F. Ungan, and I. Sökmen, Physica E 90, 214 (2017); H. Sari, F. Ungan, S. Sakiroglu, U. Yesilgul, and I. Sökmen, Optik 162, 76 (2018). 
[23] E. C. Niculescu and L. Burileanu, J. Optoelectron. Adv. Mater. 9, 2713 (2007) ; E. C. Niculescu and A. M. Radu, ibid. 9, 3598 (2007) ; F. E. López, E. Reyes-Gómez, N. Porras-Montenegro, H. S. Brandi, and L. E. Oliveira, J. Phys.: Condens. Matter 22, 045303 (2010).

[24] H. S. Brandi, A. Latgé, and L. E. Olieira, J. Appl. Phys. 92, 4209 (2002); S. Sakiroglu, D. G. Kilic, U. Yesilgul, F. Ungan, E. Kasapoglu, H. Sari, and I. Sokmen, Physica B 539, 101 (2018).

[25] See, e.g., A. Dousse, L. Lanco, J. J. Suffczyński, E. Semenova, A. Miard, A. Lemaître, I. Sagnes, C. Roblin, J. Bloch, and P. Senellart, Phys. Rev. Lett. 101, 267404 (2008); L. Schneebeli, M. Kira, and S. W. Koch, ibid. 101, 097401 (2008).

[26] B. K. Meyer, D. M. Hofmann, D. Volm, W. M. Chen, N. T. Son, and E. Janźen, Phys. Rev. B 61, 4844 (2000); W. Xu, R. A. Lewis, P. M. Koenraad, and C. J. G. M. Langerak, J. Phys.: Condens. Matter 16, 89 (2004).

[27] W. Y. Wang and W. Xu, Phys. Rev. B 86, 045307 (2012).

[28] W. Wang, B. Van Duppen, M. Van der Donck, and F. M. Peeters, Phys. Rev. B 97, 064108 (2018).

[29] W. Y. Wang, X. F. Wei, K. Lv, B. Wu, S. Chang, and J. J. Mao, Phys. Lett. A 379, 2750 (2015).

[30] R. M. O. Galvão and L. C. M. Miranda, Am. J. Phys. 51, 729 (1983).

[31] R. Gargano, A. L. A. Fonseca, and O. A. C. Nunes, Phys. Rev. A 41, 2138 (1990); R. B. Pereira, A. L. A. Fonseca, and O. A. C. Nunes, J. Phys. Soc. Jpn. 67, 4098 (1998); C. RodrígueiCateilanos and M. T. Pérez-Maldonado, Superlattices Microstruct. 27, 15 (2000); M. T. Pérez-Maldonado, C. Rodríguei-Cateilanos, and M. Sánchez-Gácita, Phys. Status Solidi B 232, 130 (2002).
[32] W. C. Henneberger, Phys. Rev. Lett. 21, 838 (1968); M. Gavrila and J. Z. Kaminski, ibid. 52, 613 (1984).

[33] C. A. S. Lima and L. C. M. Miranda, Phys. Rev. A 23, 3335 (1981); Phys. Lett. A 86, 367 (1981).

[34] Y. P. Varshni, Superlattices Microstruct. 30, 45 (2001).

[35] T. Xu, L. Yuan, and J. Fang, Physica B 404, 3445 (2009); W. Xie, Physica E 43, 1704 (2011); 43, 1411 (2011); B. Vaseghi, G. Rezaei, and T. Sajadi, Physica B 456, 171 (2015).

[36] L. C. M. Miranda, R. M. O. Galvão, and C. A. S. Lima, Phys. Rev. B 28, 5313 (1983).

[37] A. P. Jauho and K. Johnsen, Phys. Rev. Lett. 76, 4576 (1996).

[38] R. M. O. Galváo and L. C. M. Miranda, J. Phys. C: Solid State Phys. 17, L41 (1984).

[39] J. J. Sakurai, Modern Quantum Mechanics (Pearson Education, Inc., San Francisco, 1994).

[40] P. M. Platzman, Phys. Rev. 125, 1961 (1962); D. R. Cohn, D. M. Larson, and B. Lax, Phys. Rev. B 6, 1367 (1972).

[41] T. Ruf and M. Cardona, Phys. Rev. Lett. 63, 2288 (1989).

[42] F. Ehlotzky, Can. J. Phys. 63, 907 (1985); Phys. Lett. A 126, 524 (1988).

[43] P. Clauws, J. Broeckx, E. Rotsaert, and J. Vennik, Phys. Rev. B 38, 12377 (1988).

[44] F. M. Peeters, A. Matulis, M. Helm, T. Fromherz, and W. Hilber, Phys. Rev. B 48, 12008 (1993).

[45] M. Şahin, Phys. Rev. B 77, 045317 (2008); M. Şahin, F. Tek, and A. Erdinç, J. Appl. Phys. 111, 084317 (2012).

[46] J. L. Pan, Phys. Rev. B 46, 4009 (1992); L. M. Ramaniah and S. V. Nair, ibid. 47, 7132 (1993); V. G. Stoleru and E. Towe, Appl. Phys. Lett. 83, 5026 (2003). 